Published in final edited form as:

Nat Genet. 2017 November ; 49(11): 1602-1612. doi:10.1038/ng.3963.

\title{
Enhancer connectome in primary human cells identifies target genes of disease-associated DNA elements
}

\author{
Maxwell R Mumbach ${ }^{1,2,12}$, Ansuman T Satpathy ${ }^{1,3,12}$, Evan A Boyle ${ }^{1,2,12}$, Chao Dai ${ }^{1,12}$, \\ Benjamin G Gowen ${ }^{4,5}$, Seung Woo Cho ${ }^{1}$, Michelle L Nguyen ${ }^{6}$, Adam J Rubin ${ }^{7}$, Jeffrey M \\ Granja $^{1,2}$, Katelynn R Kazane ${ }^{4,5}$, Yuning Wei ${ }^{1}$, Trieu Nguyen ${ }^{8}$, Peyton G Greenside ${ }^{2}$, M Ryan \\ Corces $^{1}$, Josh Tycko ${ }^{2}$, Dimitre R Simeonov6, ${ }^{6}$, Nabeela Suliman $^{2}, \mathbf{R u i} \mathbf{L i}^{1}$, Jin Xu ${ }^{1}$, Ryan A \\ Flynn $^{1}$, Anshul Kundaje ${ }^{2}$, Paul A Khavari ${ }^{7}$, Alexander Marson ${ }^{5,6,10}$, Jacob E Corn ${ }^{4,5}$, \\ Thomas Quertermous $^{8}$, William J Greenleaf ${ }^{1,2,10,11,13}$, and Howard Y Chang ${ }^{1,7,13}$ \\ ${ }^{1}$ Center for Personal Dynamic Regulomes, Stanford University School of Medicine, Stanford, \\ California, USA \\ ${ }^{2}$ Department of Genetics, Stanford University School of Medicine, Stanford, California, USA \\ ${ }^{3}$ Department of Pathology, Stanford University School of Medicine, Stanford, California, USA \\ ${ }^{4}$ Department of Molecular and Cell Biology, University of California, Berkeley, Berkeley, California, \\ USA \\ ${ }^{5}$ Innovative Genomics Institute, University of California, Berkeley, Berkeley, California, USA \\ ${ }^{6}$ Department of Microbiology and Immunology, University of California, San Francisco, San \\ Francisco, California, USA \\ ${ }^{7}$ Program in Epithelial Biology, Stanford University School of Medicine, Stanford, California, USA \\ ${ }^{8}$ Department of Medicine, Division of Cardiovascular Medicine, Stanford University School of \\ Medicine, Stanford, California, USA \\ ${ }^{9}$ Biomedical Sciences Graduate Program, University of California, San Francisco, San Francisco, \\ California, USA \\ ${ }^{10}$ Chan Zuckerberg Biohub, San Francisco, California, USA \\ ${ }^{11}$ Department of Applied Physics, Stanford University, Stanford, California, USA
}

\footnotetext{
Reprints and permissions information is available online at http://www.nature.com/reprints/index.html

Correspondence should be addressed to H.Y.C. (howchang@ @stanford.edu) or W.J.G. (wjg@ stanford.edu).

${ }_{12}$ These authors contributed equally to this work.

${ }^{13}$ These authors jointly directed this work.

AUTHOR CONTRIBUTIONS

M.R.M., A.T.S., W.J.G., and H.Y.C. conceived the project. M.R.M., A.T.S., J.T., and R.L. performed all genomics assays with help from T.N., M.R.C., N.S., and R.A.F. A.T.S. performed all sorting for experiments. B.G.G., S.W.C., M.R.M., M.L.N., K.R.K., and D.R.S. performed all CRISPR validation experiments. E.A.B., C.D., M.R.M., and J.X. analyzed HiChIP data. J.G., A.T.S., and Y.W analyzed ATAC-seq data. A.J.R. and P.G.G. analyzed GWAS SNPs in HiChIP data. A.K., P.A.K., A.M., J.E.C., T.Q., W.J.G., and H.Y.C. guided experiments and data analysis. M.R.M., A.T.S., E.A.B., C.D., W.J.G., and H.Y.C. wrote the manuscript with input from all authors.
}

COMPETING FINANCIAL INTERESTS

The authors declare competing financial interests: details are available in the online version of the paper.

Publisher's note: Springer Nature remains neutral with regard to jurisdictional claims in published maps and institutional affiliations. 


\section{Abstract}

The challenge of linking intergenic mutations to target genes has limited molecular understanding of human diseases. Here we show that $\mathrm{H} 3 \mathrm{~K} 27 \mathrm{ac}$ HiChIP generates high-resolution contact maps of active enhancers and target genes in rare primary human $\mathrm{T}$ cell subtypes and coronary artery smooth muscle cells. Differentiation of naive T cells into $\mathrm{T}$ helper 17 cells or regulatory $\mathrm{T}$ cells creates subtype-specific enhancer-promoter interactions, specifically at regions of shared DNA accessibility. These data provide a principled means of assigning molecular functions to autoimmune and cardiovascular disease risk variants, linking hundreds of noncoding variants to putative gene targets. Target genes identified with HiChIP are further supported by CRISPR interference and activation at linked enhancers, by the presence of expression quantitative trait loci, and by allele-specific enhancer loops in patient-derived primary cells. The majority of disease-associated enhancers contact genes beyond the nearest gene in the linear genome, leading to a fourfold increase in the number of potential target genes for autoimmune and cardiovascular diseases.

Gene expression programs are intimately linked to the hierarchical organization of the genome. In mammalian cells, each chromosome is organized into hundreds of megabasesized topologically associated domains (TADs), which are conserved from early stem cells to differentiated cell types ${ }^{1}$. Within this invariant TAD scaffold, cell-type-specific enhancerpromoter interactions establish regulatory gene expression programs ${ }^{2}$. Standard methods require tens of millions of cells to obtain high-resolution interaction maps and confidently assign enhancer-promoter contacts ${ }^{3-5}$. Thus, the principles that govern enhancer-promoter conformation in disease-relevant patient samples are incompletely understood. This gap in understanding is particularly problematic for interpreting the molecular functions of inherited risk factors for common human diseases, which reside in intergenic enhancers or other noncoding DNA features in up to $90 \%$ of cases $^{6-9}$. Such disease-relevant enhancers may not influence the expression of the nearest gene (often reported as the default target in the literature) and may instead act in a cell-type-specific manner on distant target genes residing up to hundreds of kilobases away ${ }^{2,10-14}$. Recently, systematic perturbations of regulatory elements in select gene loci have shown that the effects of individual regulatory elements on gene activity can be predicted from the combination of (i) enhancer activity (marked by histone $\mathrm{H} 3$ lysine 27 acetylation (H3K27ac) level) and (ii) enhancer-target looping ${ }^{5,15}$. Here we leverage this insight to capture the combination of these two types of information across the genome in a single assay, mapping the enhancer connectome in disease-relevant primary human cells.

\section{RESULTS}

\section{H3K27ac HiChIP identifies functional enhancer interactions}

We recently developed HiChIP, a method for sensitive and efficient analysis of proteincentric chromosome conformation ${ }^{16}$. Cohesin HiChIP in GM12878 cells identified similar numbers of loops as in situ Hi-C $(\sim 10,000)$ with high correlation $(R=0.83)$, demonstrating that HiChIP captures loops with high sensitivity and specificity. Here we evaluated the enhancer- and promoter-associated mark $\mathrm{H} 3 \mathrm{~K} 27 \mathrm{ac}^{17-19}$ as a candidate factor to selectively 
interrogate enhancer-promoter interactions across the genome. We performed H3K27ac HiChIP in mouse embryonic stem (ES) cells to compare to cohesin HiChIP (Supplementary Fig. 1a and Supplementary Table 1) ${ }^{16}$. 3,552 of the 4,191 H3K27ac HiChIP loops in mouse ES cells were also identified by cohesin HiChIP. The H3K27ac-biased loops ( $\log _{2}$ (fold change) > 1) spanned shorter distances than the cohesin-biased loops and were enriched for H3K27ac ChIP-seq peaks (78.9\%; Supplementary Fig. 1b-f and Supplementary Table 2). Moreover, systematic titration of input material showed that $\mathrm{H} 3 \mathrm{~K} 27 \mathrm{ac}$ HiChIP retained high signal fidelity and reproducibility when using from 25 million to 50,000 cells as input material (loop signal correlation $r=0.918$; Supplementary Figs. 2 and 3). Therefore, H3K27ac HiChIP identifies high-confidence chromatin loops focused around enhancer interactions from limited cell numbers.

To capture (i) conformational change during $\mathrm{T}$ cell differentiation and (ii) cell-type-specific chromatin contacts of risk variants for autoimmune diseases in protective and pathogenic $\mathrm{T}$ cell types, we performed $\mathrm{H} 3 \mathrm{~K} 27 \mathrm{ac}$ HiChIP on primary human naive $\mathrm{T}$ cells $\left(\mathrm{CD} 4^{+} \mathrm{CD} 45 \mathrm{RA}\right.$ $\left.{ }^{+} \mathrm{CD} 25^{-} \mathrm{CD} 127^{\text {hi }}\right)$, regulatory $\mathrm{T}\left(\mathrm{T}_{\text {reg }}\right)$ cells $\left(\mathrm{CD} 4{ }^{+} \mathrm{CD} 25^{+} \mathrm{CD} 127^{\mathrm{lo}}\right)$, and $\mathrm{T}$ helper $17\left(\mathrm{~T}_{\mathrm{H}} 17\right)$ cells (CD4+CD45RA ${ }^{-} \mathrm{CD} 25^{-} \mathrm{CD} 127^{\mathrm{hi}} \mathrm{CCR}^{+} \mathrm{CXCR}^{-}$) directly isolated from donors (Fig. 1a,b and Supplementary Fig. $4 a)^{20,21} \cdot \mathrm{T}_{\mathrm{H}} 17$ cells were sorted to include autoimmune disease-relevant pathogenic $\mathrm{T}_{\mathrm{H}} 17$ cells and to exclude follicular helper $\mathrm{T}$ cells with a distinct surface phenotype and immune function (Supplementary Fig. 4a) ${ }^{22-24}$. Peripheral blood $\mathrm{CD}^{+} \mathrm{T}$ cells were obtained from three healthy subjects, isolated by FACS, and subjected to H3K27ac HiChIP. The HiChIP libraries from each subset were high quality; greater than $40 \%$ of the reads represented unique paired-end tags (PETs) (Supplementary Fig. 4b-d and Supplementary Table 1). Furthermore, the libraries exhibited high 1D signal enrichment at enhancers and promoters and globally recapitulated publically available H3K27ac ChIP-seq data sets (74.7\% overlap of ChIP-seq and 1D HiChIP peaks; Fig. 1c) ${ }^{25}$. Inspection of the interaction matrix at progressively higher resolution showed chromatin compartments, TADs, and focal loops, as previously reported in high-resolution $\mathrm{Hi}-\mathrm{C}$ and HiChIP analyses from cell lines (Fig. 1b) ${ }^{4,16}$. Notably, H3K27ac HiChIP maps were capable of identifying focal interactions at $1-\mathrm{kb}$ resolution, which is comparable to the resolution for in situ Hi-C maps generated from 100-fold more cells and sequenced to 13-fold greater $\operatorname{depth}^{4}$ (Fig. 1b).

Previous saturation perturbation screens demonstrated that functional enhancers can be identified by integrating H3K27ac ChIP-seq signal with chromosome conformation contact strength (Hi-C) $)^{5}$. Because H3K27ac HiChIP combines these two components into one assay, we reasoned that HiChIP signal, which we term enhancer interaction signal (EIS), should identify functional regulatory elements. To validate this prediction, we first generated H3K27ac HiChIP maps in a chronic myelogenous leukemia cell line (K562) as a direct comparison to published high-resolution CRISPR interference (CRISPRi) screens ${ }^{5}$. We then examined the 3D enhancer landscape of the $M Y C$ and GATA1 loci using virtual 4C (v4C) analysis, where a specific genomic position is set as an anchor viewpoint and all interactions occurring with that anchor are visualized in $2 \mathrm{D}^{16}$. $\mathrm{v} 4 \mathrm{C}$ analysis of the $M Y C$ promoter demonstrated that EIS in K562 cells captured all functional enhancers identified in the CRISPRi screen (Fig. 2a). Analysis of the GATA1 locus demonstrated a similar agreement between the two methods (Fig. 2b). Quantitatively, the EIS in K562 cells was significantly 
correlated with CRISPRi score in the same cell type, whereas the EIS in GM12878 (GM; B cell lymphoblast) cells was not correlated with the K562 CRISPRi score (Spearman's $\rho=$ 0.332 and $0.145 ; P=9.25 \times 10^{-5}$ and 0.1246 ; Fig. $2 c$ ).

We found the enhancer landscapes of the $M Y C$ promoter to be highly cell type specific. v4C analysis of the $M Y C$ promoter in GM and My-La (CD4 ${ }^{+} \mathrm{T}$ cell leukemia) cells showed dramatically different regulatory interactions with the promoter as compared to K562 cells (Fig. 2d). To validate EIS specificity, we performed CRISPRi experiments in GM cells using single guide RNAs (sgRNAs) targeting enhancers identified in either GM or My-La HiChIP maps, as well as a positive-control sgRNA targeting the $M Y C$ promoter and a negativecontrol sgRNA targeting lambda phage sequence (Fig. 2e). As expected, we found that simultaneous CRISPRi perturbation of GM enhancers, but not My-La enhancers, impacted $M Y C$ expression and cell growth in GM cells (Fig. 2e).

Finally, we focused on the $C D 69$ locus, where a high-resolution CRISPR activation (CRISPRa) screen identified three enhancers upstream of the transcription start site (TSS) ${ }^{26}$. These sites were also identified by H3K27ac HiChIP in naive T cells. Moreover, HiChIP identified four additional distal enhancers that were outside the region spanned by the sgRNA tiling array (Fig. 2f and Supplementary Fig. 5). To functionally validate these new enhancers, we performed CRISPRa experiments in Jurkat cells with sgRNAs targeting these enhancers, the $C D 69$ promoter, and the $K L R F 2$ promoter as a locus negative control, as well as a non-human-genome-targeting negative control. We observed a significant increase in CD69 RNA and protein levels in the four HiChIP enhancers as compared to the negative controls (Fig. 2g and Supplementary Fig. 5). Interestingly, two of the four new enhancers identified were within the promoter regions of distant genes. These findings are in line with previous reports that identified widespread distal gene regulatory functions of promoters across the genome ${ }^{27,28}$. Altogether, these results suggest that H3K27ac HiChIP EIS identifies functional regulatory elements and that enhancers that regulate a gene of interest can differ significantly between cell types.

\section{Landscape of enhancer interactions in primary $T$ cells}

We examined global features of the enhancer connectome associated with cellular differentiation from naive $\mathrm{T}$ cells into either $\mathrm{T}_{\mathrm{H}} 17$ cells or $\mathrm{T}_{\text {reg }}$ cells. We identified a total of 10,706 high-confidence loops in the union set of the three cell types (Supplementary Table 2). Analysis of loop read support between biological replicates demonstrated high reproducibility (Supplementary Fig. 4 c), and $\sim 91 \%$ of loop anchors were associated with either a promoter or an enhancer ${ }^{29}$, as expected, with a median distance of $130 \mathrm{~kb}$ (Supplementary Fig. 6a,b). Notably, high-resolution enhancer-promoter connectivity maps identified several features that could not be discerned from 1D epigenomic data (that is, H3K27ac ChIP-seq or assay for transposase-accessible chromatin using sequencing, ATACseq; Fig. 3a). These features included (i) 'enhancer skipping': enhancers that had stronger EIS with a more distal target promoter; (ii) higher-order structures such as 'enhancer cliques' (related to loop cliques ${ }^{30}$ ): multiple regulatory elements that had strong EIS with a single target promoter; (iii) promoter-promoter interactions ${ }^{13,31}$; and (iv) 'enhancer 
switching': enhancers that exhibited differential EIS with a target promoter in a cell-typespecific manner (Fig. 3a).

We found that EIS contacts were very cell type specific. After quantile-quantile normalization of contact reads at high-confidence loops (correcting for false positives caused by 1D fragment visibility; Supplementary Note), we focused on the top and bottom 5\% of EIS ranked by cell type bias for each pairwise comparison (Supplementary Figs. 6c-g and 7, and Supplementary Tables 3 and 4). Cell-type-specific enhancer loop anchors identified genes encoding canonical $\mathrm{T}$ cell subtype transcription factors and effector molecules (Fig. $3 \mathrm{~b}$ and Supplementary Figs. 8 and 9). Deeper v4C analysis of shared and cell-type-specific loci pinpointed regulatory elements interacting with each gene promoter of interest as well as local conformational landscape changes (Supplementary Figs. 8 and 9). Transcription factor motifs located within cell-type-specific loop anchors were enriched for transcription factors known to drive $\mathrm{T}$ cell subtype differentiation and nominated new transcription factors involved in regulation (Fig. 3c). Furthermore, cell type EIS bias was associated with differential expression of genes located within corresponding EIS anchors for the same cell type (naive to $\mathrm{T}_{\mathrm{H}} 17$ : Spearman's $\rho=0.242$ and $P=4 \times 10^{-15}$; naive to $\mathrm{T}_{\text {reg: }}$ : Spearman's $\rho=$ 0.207 and $P=2 \times 10^{-11}$ ); Fig. $3 \mathrm{~d}$ ).

Cell-type-specific EIS may be driven by cell-type-specific enhancer activation (based on H3K27ac ChIP-seq) or stable enhancer activation with cell-type-specific looping (Hi-C) in a gene-specific manner. We first examined H3K27ac ChIP-seq signal at differential EIS anchors and found that many biased H3K27ac HiChIP interactions also exhibited biased ChIP-seq signal, as expected. 58.5\% of naive T cell-biased loops contained at least one naive T cell-biased ChIP-seq peak ( $\log _{2}$ (fold change) $>1$ ) located on the anchors. Similarly, $66.7 \%$ of $\mathrm{T}_{\mathrm{H}} 17$ cell-biased and $67.8 \%$ of $\mathrm{T}_{\text {reg }}$ cell-biased interaction anchors were cell type specific in 1D (Supplementary Fig. 10a). Therefore, while on average $~ 64 \%$ of the differential EIS corresponds to changes in 1D data, $236 \%$ is likely also driven by changes in 3D chromatin loop strength. To further assess the contribution of cell-typespecific 3D signal to EIS, we examined HiChIP 1D signal at differential EIS anchors. We found that HiChIP 1D signal correlated better with ChIP-seq signal than EIS, with a higher likelihood of differential ChIP-seq signal overlapping differential HiChIP 1D signal as compared to 3D signal, suggesting that EIS bias is in part driven by 3D changes (Supplementary Fig. 10b).

We asked whether the integration of reference cell line Hi-C data with primary $\mathrm{T}$ cell H3K27ac ChIP-seq data could recapitulate HiChIP EIS in primary T cells. We binned GM Hi-C loops with increasing primary $\mathrm{T}$ cell ChIP-seq signal at loop anchors and then determined the overlap of loops in each bin with loops derived from H3K27ac HiChIP. As expected, increased ChIP-seq signal at the Hi-C anchors led to increased overlap with the HiChIP loops. However, the overlap was lower in all $\mathrm{T}$ cell subtypes as compared to the same analysis performed using GM HiChIP data. These observations demonstrate that celltype-specific 3D interactions can impact EIS independently of differences in 1D ChIP-seq signal (Supplementary Fig. 10c). Similarly, previously generated enhancer-promoter maps obtained from bulk $\mathrm{T}$ cells did not identify $\mathrm{T}$ cell subtype-specific interactions obtained using $\mathrm{H} 3 \mathrm{~K} 27 \mathrm{ac} \mathrm{HiChIP.} \mathrm{To} \mathrm{assess} \mathrm{the} \mathrm{unique} \mathrm{information} \mathrm{obtained} \mathrm{through} \mathrm{cell-type-}$ 
specific interaction maps, we compared promoter-capture Hi-C maps ${ }^{14}$ in bulk $\mathrm{CD} 4^{+} \mathrm{T}$ cells to $\mathrm{H} 3 \mathrm{~K} 27 \mathrm{ac}$ HiChIP maps in naive $\mathrm{T}, \mathrm{T}_{\mathrm{H}} 17$, and $\mathrm{T}_{\text {reg }}$ cells. Strikingly, the most cell-typespecific loops in $\mathrm{T}_{\mathrm{H}} 17$ and $\mathrm{T}_{\text {reg }}$ cells (16-fold enriched) demonstrated a low discovery rate in promoter-capture Hi-C T cells (11.83\% in 415 loops and $13.83 \%$ in 373 loops, respectively; Supplementary Fig. 10d). Many of these subset-specific interactions included genomic loci encoding functionally important effector genes, such as LRRC32. The LRRC32 locus contained $\mathrm{T}_{\text {reg }}$-specific loops that were neither visualized in HiChIP maps from naive $\mathrm{T}$ or $\mathrm{T}_{\mathrm{H}} 17$ cells nor in bulk CD4+ promoter-capture Hi-C maps (Supplementary Fig. 10e). Because primary human $\mathrm{T}_{\mathrm{H}} 17$ and $\mathrm{T}_{\text {reg }}$ cells are present in human blood at low frequencies, it would also be challenging to generate subset-specific promoter-capture Hi-C maps with published promoter-capture Hi-C protocols. In summary, EIS is derived from a combination of 1D ChIP-seq signal and 3D interaction signal and cannot be accurately predicted from 3D maps in reference cell lines or unsorted primary cell data sets.

Cell-type-specific EIS can occur at sites of shared chromatin accessibility. Paired chromatin accessibility profiles from ATAC-seq ${ }^{32}$ for each T cell subset showed that most cell-typespecific loop anchors had equivalent chromatin accessibility across all three cell types (Fig. $3 \mathrm{e}-\mathrm{g}$ ). To illustrate this finding, we examined the $\mathrm{BACH} 2$ promoter, which exhibited shared chromatin accessibility at enhancers but increased EIS in naive T cells (Fig. 3e). Globally,

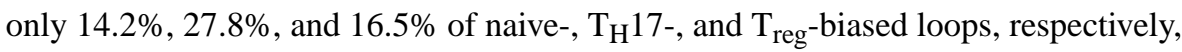
contained at least one biased ATAC-seq peak ( $\log _{2}$ (fold change) $>1$ ) located on the anchors. Furthermore, the majority of cell-type-specific transcription factor motifs were observed in shared ATAC-seq peaks within differential interactions, highlighting the notion that these regions are functioning in $\mathrm{T}$ cell differentiation (Fig. 3f,g). Altogether, these results suggest that, in highly related—yet functionally distinct—cell types, a portion of transcriptional control is achieved through differential chromosome looping, rather than differential chromatin accessibility. This finding is consistent with previous studies that demonstrated that $\mathrm{T}$ cell subset-specific transcription factors, such as FOXP3, act predominantly at pre-accessible chromatin sites to establish subset-specific gene expression $^{33}$.

\section{Enhancer interactions link disease variants to target genes}

The high specificity of EIS enabled us to identify putative target genes of autoimmune disease risk loci in functionally relevant $\mathrm{T}$ cell subsets. To achieve this, we used a previously described list of putatively causal variants associated with 21 autoimmune diseases, known as PICS SNPs, which were fine-mapped on the basis of dense genotyping data ${ }^{25}$. We determined that PICS autoimmune disease-associated SNPs were significantly enriched in T cell loop anchors, with variants for specific autoimmune diseases showing greater than fivefold enrichment as compared to a shuffled control loop set (Supplementary Fig. 11). Next, we constructed a set of all possible connections between autoim-mune disease risk SNPs and TSSs within $1 \mathrm{Mb}$ and measured the EIS for each SNP-TSS pair (Fig. 4a). We aggregated these signals to determine the overall interaction activity in each $\mathrm{T}$ cell subtype for each disease (Fig. 4b). We observed high interaction strength enrichments and cell type specificity for autoimmune disease-associated SNPs, but low enrichment and cell type specificity for variants associated with non-immune traits (Fig. 4b). To further visualize 
HiChIP bias in shared or differential enhancers, we analyzed SNP-TSS interactions grouped by their presence near H3K27ac ChIP-seq peaks (Supplementary Fig. 12a,b). We observed a large number of active SNP-TSS pairs that were present in regulatory regions that were shared by $\mathrm{T}$ effector cell types ( $\mathrm{T}_{\text {reg }}$ and $\mathrm{T}_{\mathrm{H}} 17$ cells), whereas relatively less EIS was observed for SNPs located in cell-type-specific enhancers, supporting the concept that many autoimmune disease variants impact common $\mathrm{T}$ cell effector/activation pathways ${ }^{25,34}$.

Notably, SNPs present in enhancers shared by all three cell types could still be distinguished by HiChIP bias (Supplementary Fig. 12a,b). For example, although we could not detect cell type bias at risk loci for alopecia areata using H3K27ac ChIP-seq data (Supplementary Fig. 12a,b and ref. 3), H3K27ac HiChIP identified increased SNP-TSS activity in $\mathrm{T}_{\text {reg }}$ cells at shared $\mathrm{T}$ cell enhancers, consistent with several studies identifying the crucial role of this cell type in disease pathogenesis ${ }^{35}$. Of note, autoimmune disease signal enrichments were not readily apparent from 1D H3K27ac ChIP-seq peaks, aggregated ChIP-seq signal within the TAD containing the SNP, or cell line H3K27ac HiChIP data sets (Fig. 4b and Supplementary Fig. 12c). Therefore, examining 3D disease variant interactions may capture cell type biases more robustly than 1D epigenomic data. Finally, to validate our findings with an orthogonal data set, we performed SNP-TSS EIS analysis on an overlapping set of autoimmune disease-associated SNPs obtained from the National Heart, Lung, and Blood Institute (NHLBI) GRASP catalog. We observed a similar pattern of enrichment for T cell subset-specific HiChIP signal in disease-associated variants (Supplementary Fig. 12d).

We leveraged HiChIP to identify potential gene targets of intergenic SNPs, which have classically been paired to the nearest neighboring gene. We overlapped the SNP-TSS pairs with loops to call a discrete set of target pairs. We then performed differential analysis on the SNP-TSS loops to ascertain bias for specific T cell subsets (Fig. 4c and Supplementary Table 5). Examples of biased SNP-TSS pairs included FOXO1 in naive T cells (rs9603754), $B A T F$ (rs2300604) in memory T cells, CTLA4 (rs10186048) in $\mathrm{T}_{\text {reg }}$ cells, and IL2 (rs7664452) in $\mathrm{T}_{\mathrm{H}} 17$ cells (Fig. $4 \mathrm{c}$ and Supplementary Table 5). Next, we sought to characterize the connectivity landscape of the SNP-TSS loops. We identified an average of 1.75 gene targets per autoimmune disease-associated SNP (ranging from 0 to over 10 target genes), whereas variants for non-immune traits did not demonstrate an increase in the number of targets ( 0.33 genes per SNP; Supplementary Fig. 12e). For 684 autoimmune disease intergenic SNPs, we identified a total of 2,597 HiChIP target genes, representing a fourfold increase in the number of target genes for known disease-associated SNPs (Fig. 4d). Only $367(\sim 14 \%)$ of all targets were the nearest gene to the SNP, while approximately $\sim 86 \%$ of SNPs skipped at least one gene to reach a predicted target TSS (Supplementary Fig. 12e). Furthermore, approximately $\sim 45 \%$ of SNP to HiChIP target interactions had increased signal as compared to the interaction between the same SNP and the nearest gene, despite distance biases.

\section{Target gene validation by eQTL and CRISPRi}

HiChIP enhancer-target gene interactions can be validated using previously identified point mutations that alter expression at distantly located genes in T cells-that is, expression quantitative trait loci (eQTLs) ${ }^{36}$. For example, the celiac disease-associated SNP rs2058660 impacts expression of the inflammatory cytokine receptor genes IL18RAP, IL18RI, 
IL1RL1, and IL1RL2, which are known regulators of intestinal T cell differentiation and response $^{37}$. HiChIP EIS showed contacts between rs2058660 and the promoters of each of these predicted target genes (Supplementary Fig. 13a). Similarly, the Crohn's disease risk variant rs6890268 and the multiple sclerosis risk variant rs12946510 impact the expression of PTGER4 and IKZF3, respectively, and H3K27ac HiChIP also demonstrated clear contacts between these SNPs and their predicted promoters (Supplementary Fig. 13a). Globally, HiChIP contact signal was increased in eQTLs in T cells as compared to a distance-matched background loop set $\left(P<2.2 \times 10^{-16}\right.$; Fig. 4 e) or to eQTLs identified in an unrelated cell type (liver; $P<2.2 \times 10^{-16}$ ). The overlap of HiChIP and eQTL loci provides support for chromosome interactions as a physical basis for distal eQTLs ${ }^{10-12}$ and further validates the HiChIP approach to assign enhancer-target gene relationships.

We next sought to directly validate HiChIP SNP-gene target interactions using CRISPRi in My-La cells. First, we focused on three loci of interest in primary T cells and then confirmed that the SNP-TSS loops were also present in My-La cells (Fig. 4f and Supplementary Fig. 13b). We then targeted sgRNAs to these SNP-containing enhancers, as well as positivecontrol sgRNAs to the HiChIP target gene promoters and a non-human-genome-targeting negative control. As expected, we observed a significant reduction in RNA levels for the HiChIP target genes upon CRISPRi of the corresponding SNP-containing enhancers (Fig. $4 f)$.

\section{Fine-mapping of disease-associated DNA variants}

As SNP-TSS HiChIP signal is capable of identifying the target genes of candidate SNPs, we asked whether TSS-SNP HiChIP signals could also be used to nominate functional causal variants within haplotype blocks in a reciprocal manner. We first performed a proof-ofprinciple analysis using fine-mapped SNPs associated with inflammatory bowel disease $(\mathrm{IBD})^{38}$ or type 1 diabetes (T1D) ${ }^{39}$ as well as high-confidence PICS SNPs and examined EIS from putatively causal SNPs to all gene promoters within $300 \mathrm{~kb}$. EIS from putatively causal SNPs to gene promoters was significantly higher than EIS from a distance-matched set of SNPs within the same linkage disequilibrium (LD; $r^{2} \geq 0.8$ ) block to gene promoters $\left(P=2.4 \times 10^{-15}, 8.7 \times 10^{-8}\right.$, and $3.9 \times 10^{-3}$ for IBD fine-mapped SNPs, T1D fine-mapped SNPs, and high-confidence PICS SNPs, respectively; Fig. 5a and Supplementary Fig. 14a). Next, we assessed the fine-mapping ability of HiChIP EIS at individual loci of interest. We focused on IBD- and multiple sclerosis-associated SNPs neighboring the PTGER4 and $S A T B 1$ loci and performed $\mathrm{v} 4 \mathrm{C}$ analysis anchored at the gene promoters. We calculated EIS signal at 1-kb resolution and identified specific regions within the LD blocks that contained the highest EIS to the target promoters, positioning the likely causal SNPs within these regions (Fig. 5b and Supplementary Fig. 14b). For example, at the PTGER4 locus (Fig. 5b), the $\sim 160$-kb genomic interval spanned by LD SNPs in association with Crohn's disease was refined to two bins of $3 \mathrm{~kb}$ and $4 \mathrm{~kb}$, which both contained PICS SNPs.

We asked whether complex disease-associated loci containing more than one gene could be fine-mapped using HiChIP. We focused on two disease-associated enhancers in between the STAT1 and STAT4 gene promoters (Fig. 5c). These two genes encode transcription factors with distinct roles in immune regulation. Signal transducer and activator of transcription 1 
(STAT1) is critical for type I interferon (IFN) and IFN- $\gamma$ signaling, whereas STAT4 induces $\mathrm{T}_{\mathrm{H}} 1$ differentiation and IFN- $\gamma$ expression ${ }^{40}$. We investigated bias of these enhancers to STAT1 and STAT4 and found that, despite comparable linear distances and 1D signals at the promoters, the enhancers were biased to interact with STAT4. Next, we fine-mapped the disease-associated SNPs within this locus using 1-kb-resolution EIS from the STAT4 promoter and narrowed down candidate functional variants within the two enhancers (Fig. 5c). In summary, HiChIP EIS can nominate functional causal variants within haplotype blocks, and two-way analysis of target gene identification from an enhancer of interest and high-resolution interaction maps of that enhancer with its target gene can be used to finemap disease-associated loci containing several candidate genes.

\section{Allelic target gene bias of cardiovascular disease variants}

Finally, we asked whether this approach could be applied broadly to other categories of human disease and whether we could directly test SNP-TSS associations using allelespecific HiChIP. We generated high-resolution enhancer-promoter maps from primary human coronary artery smooth muscle cells (HCASMCs), which can be used to inform on variants linked to cardiovascular diseases ${ }^{41}$. First, to validate cell type specificity, we examined the gene promoter for TCF21, a transcription factor required for the differentiation of HCASMCs ${ }^{42}$, and observed enrichment of HCASMC EIS relative to naive T cells (Fig. 6a). We next examined the 9p21.3 locus, which harbors risk associations with several cardiovascular disorders ${ }^{43-45}$. We found that the promoters of all three genes in the locus interacted with one another and with CAD-variant-containing enhancers located approximately $100 \mathrm{~kb}$ upstream of the $C D K N 2 B$ promoter (Supplementary Fig. 15). We then generated SNP-TSS target lists using CAD-associated SNPs identified in the CARDIoGRAMplusC4D study ${ }^{46}$. We again performed differential analysis on the SNP-TSS loops to ascertain bias for HCASMCs versus naive T cells (Fig. 6b). Overall, $75.1 \%$ of HCASMC-biased SNP-TSS pairs involved CAD-associated SNPs, whereas only $5.5 \%$ of naive T cell-biased SNP-TSS pairs were CAD SNP-TSS loops. Next, we examined the connectivity of the HCASMC SNP-TSS contacts and identified 1,062 gene targets, of which only $120(\sim 11 \%)$ mapped to the nearest gene. Furthermore, approximately $89 \%$ skipped at least one gene to reach a predicted target TSS, and $64 \%$ of SNPs were mapped to more than a single gene target.

We took advantage of genome phasing information in HCASMCs to measure enhancerpromoter interactions at allele-specific CAD-associated SNPs, allowing us to examine the functional consequence of a risk variant as compared to its alternative allele in the same nucleus. First, $4.2 \%$ of high-confidence loops in HCASMCs with no observed mapping bias in the anchors exhibited significant allelic bias (FDR < 0.05; Fig. 6c), consistent with the frequency of allelic imbalance of RNA expression and prior evidence of allele-specific regulation of specific enhancer-promoter interactions ${ }^{47,48}$. We leveraged this global enhancer-promoter allelic bias to examine the effect of a risk-associated variant as compared to its control alternative allele for a set of CAD-associated SNP-target gene pairs (Fig. 6d) ${ }^{49}$. We found that many risk alleles disrupted enhancer-target gene interactions, but a subset of pathogenic SNPs increased enhancer-target gene interaction. At CAD risk variant rs1537373 in the 9p21.3 locus, the risk allele (T) showed increased EIS to the 
$C D K N 2 A$ promoter as well as an additional enhancer within the long noncoding RNA (lncRNA) ANRIL gene relative to the reference allele (G) (Fig. 6e). We further observed increased EIS of the CAD risk variant rs4562997 to an additional SMAD3 enhancer $10 \mathrm{~kb}$ downstream of the TSS (Fig. 6e). The ability to resolve enhancer connectomes of the risk and reference alleles in the same nucleus demonstrates that the mutated base in the risk allele suffices to alter enhancer looping in cis in disease-relevant primary cells.

\section{DISCUSSION}

Here we developed an approach to define the high-resolution landscape of enhancerpromoter regulation in primary human cells. We find that enhancer-promoter contacts are highly dynamic in related cell types and often involve genomic elements with shared accessibility. Accordingly, many complex features of the 3D enhancer connectome cannot simply be predicted from 1D data, demonstrating that mapping conformation in primary cells can identify new regulatory connections underlying gene function in human disease. We take advantage of this principle to chart the connectivity of autoimmune and cardiovascular disease genome-wide association study (GWAS)-identified SNPs and link SNPs to hundreds of potential target genes. Although non-genic SNPs have previously been paired with their closest neighboring gene, we find that the majority of these variants can engage in long-distance interactions, including skipping several promoters to predicted target genes, connecting to multiple genes, or acting in concert with enhancer cliques to contact a single gene. Further use of this approach will help to clarify hidden mechanisms of human disease that are driven by genetic perturbations in non-protein-coding DNA elements, which can now be linked to their cognate gene targets in primary cells.

\section{ONLINE METHODS}

\section{Human subjects}

This study was approved by the Stanford University Administrative Panels on Human Subjects in Medical Research, and written informed consent was obtained from all participants.

\section{Cell culture and primary $\mathrm{T}$ cell isolation}

Mouse ES cells (v6.5, Novus Biologicals, NBP1-41162) were cultured in Knockout DMEM (Gibco) supplemented with 15\% FBS and leukemia inhibitory factor (LIF; Millipore) to $80 \%$ confluence. GM12878 (Coriell), Jurkat, and My-La $\left(\mathrm{CD}^{+}\right)$cells (ATCC) were grown in RPMI 1640 (Gibco) supplemented with 15\% FBS to a concentration of 500,000 to 1 million cells $/ \mathrm{ml}$. Normal donor human peripheral blood cells were obtained fresh from AllCells. CD4 ${ }^{+} \mathrm{T}$ cells were enriched from peripheral blood using RosetteSep Human CD4 ${ }^{+}$ T Cell Enrichment Cocktail (StemCell Technology). For $\mathrm{CD}^{+}{ }^{+} \mathrm{T}$ helper cell subtypes, naive T cells were sorted as $\mathrm{CD}^{+} \mathrm{CD} 25^{-} \mathrm{CD} 45 \mathrm{RA}^{+}$cells, $\mathrm{T}_{\mathrm{H}} 17$ cells were sorted as $\mathrm{CD} 4+\mathrm{CD} 25-$ $\mathrm{CD} 4 \mathrm{RA}^{-} \mathrm{CCR}^{+} \mathrm{CXCR}^{-}$cells, and $\mathrm{T}_{\text {reg }}$ cells were sorted as $\mathrm{CD}^{+} \mathrm{CD} 25^{+} \mathrm{CD} 127^{\mathrm{lo}}$ cells. Antibodies used for FACS included the following: PerCP/Cy5.5 anti-CD45RA (BioLegend, 304122), Brilliant Violet 510 anti-CD127 (BioLegend, 351331), APC/Cy7 anti-CD4 (BioLegend, 344616), PE anti-CCR6 (BioLegend, 353410), FITC anti-CD25 (BioLegend, 
302603), Brilliant Violet 421 anti-CXCR3 (BioLegend, 353715), and BB515 anti-CXCR5 (BD Biosciences, 564625). For HiChIP experiments, 500,000 to 1 million cells were sorted into RPMI medium supplemented with $10 \%$ FCS. For ATAC-seq experiments, 55,000 cells were sorted into RPMI medium supplemented with 10\% FCS. Post-sort purities of $>95 \%$ were confirmed by flow cytometry for each sample.

A primary HCASMC line derived from a normal human donor heart was purchased from Cell Applications (350-05A) and cultured in smooth muscle growth medium (Lonza, CC-3182) supplemented with hEGF, insulin, hFGF-b, and 5\% FBS. Cells were grown according to Lonza's instructions.

\section{Cell fixation}

Detached cell lines or sorted $\mathrm{CD} 4^{+} \mathrm{T}$ cells were pelleted and resus-pended in fresh $1 \%$ formaldehyde (Thermo Fisher) at a volume of $1 \mathrm{ml}$ of formaldehyde per 1 million cells. Cells were incubated at room temperature for $10 \mathrm{~min}$ with rotation. Glycine was added at a final concentration of $125 \mathrm{mM}$ to quench the formaldehyde, and cells were incubated at room temperature for $5 \mathrm{~min}$ with rotation. Finally, cells were pelleted and washed with PBS, pelleted again, and stored at $-80{ }^{\circ} \mathrm{C}$ or immediately taken into the HiChIP protocol.

\section{HiChIP}

The HiChIP protocol was performed as previously described, using antibody to H3K27ac (Abcam, ab4729) or CTCF (Abcam, ab70303) ${ }^{16}$ with the following modifications. For primary $\mathrm{T}$ cells, we performed HiChIP on as many cells as we could obtain from a blood donation-approximately 500,000 to 1 million cells per T cell subtype per replicate. We performed 2 min of sonication, did not carry out Protein A bead preclearing, used $4 \mu \mathrm{g}$ of antibody to H3K27ac (Abcam, ab4729), and captured the chromatin-antibody complex with $34 \mu \mathrm{l}$ of Protein A beads (Thermo Fisher). Qubit quantification following ChIP ranged from 5-25 ng depending on the cell type and amount of starting material. The amount of Tn5 used and number of PCR cycles performed were based on the post-ChIP Qubit amounts, as previously described ${ }^{16}$.

Twenty-five million cell line libraries were generated as previously described ${ }^{16}$. For mouse ES cell samples with low cell numbers, we performed 2 min of sonication and did not carry out Protein A bead preclearing. Either $4 \mu \mathrm{g}$ or $2 \mu \mathrm{g}$ of antibody to H3K27ac (Abcam, ab4729) was used for ChIP in 500,000 or 100,000/50,000 cells, respectively, and the chromatin-antibody complex was captured with 34 (500,000 cells) or 20 (100,000/50,000 cells) $\mu \mathrm{l}$ of Protein A beads. Post-ChIP Qubit quantification for the 25 million cell samples was approximately $1.5 \mu \mathrm{g}$. For lower cell numbers, quantification was 30,10 , and $5 \mathrm{ng}$ for $500,000,100,000$, and 50,000 cells, respectively. The amount of Tn5 used and the number of PCR cycles performed were based on the post-ChIP Qubit amounts, as previously described.

HiChIP samples were size selected by PAGE purification (300-700 bp) for effective pairedend tag mapping and were therefore removed of all primer contamination that would contribute to recently reported 'index switching' on the Illumina HiSeq 4000 sequencer ${ }^{50}$. All libraries were sequenced on the Illumina HiSeq 4000 instrument to an average depth of 500-600 million total reads. 


\section{HiChIP data processing}

HiChIP paired-end reads were aligned to the hg 19 or mm9 genome using the HiC-Pro pipeline ${ }^{51}$. Default settings were used to remove duplicate reads, assign reads to MboI restriction fragments, filter for valid interactions, and generate binned interaction matrices. HiC-Pro filtered reads were then processed into a .hic file using the hicpro2juicebox function. The Juicer pipeline HiCCUPS tool was used to identify high-confidence loops ${ }^{4}$ using the same parameters as for the GM12878 in situ Hi-C map: hiccups -m 500 -r 5000,10000 -f 0.1,0.1 -p 4,2 -i 7,5 -d 20000,20000 .hic_input HiCCUPS_output. For T cell Juicer loops, performing the default Juicer calls resulted in a high rate of false positives upon visual inspection of the interaction matrix. We therefore called loops with the same HiCCUPS parameters in two biological replicates for each T cell subtype and then filtered loops for those that were reproducibly called in both replicates. In addition, we removed all loops greater than $1 \mathrm{Mb}$ in length.

1D signal enrichment and peak calling were generated from the HiC-Pro filtered contacts file. Intrachromosomal contacts were filtered, and both anchors were extended by $75 \mathrm{bp}$. The combined bed file containing both anchors was then used to generate bigwigs for visualization in the WashU Epigenome Browser or call peaks using MACS2.

Allele-specific HiChIP data processing was achieved using HiC-Pro's allele-specific analysis features ${ }^{51}$. First, HCASMC phasing data ${ }^{41}$ were used to mask the hg 19 genome and make indexes. HiC-Pro settings were similar to those described above, with the exception that reads were aligned to the masked genome and then assigned to a specific allele on the basis of phasing data.

\section{Interaction matrices and virtual $4 \mathrm{C}$ visualization}

HiChIP interaction maps were generated with Juicebox using KR matrix balancing and visualized using Juicebox software at 500-kb, 25-kb, 10-kb, and 5-kb resolution as indicated in each analysis ${ }^{4}$. For $1-\mathrm{kb}$ profiles, raw matrix counts were visualized in Java TreeView.

v4C plots were generated from dumped matrices generated with Juicebox. The Juicebox tools dump command was used to extract the chromosome of interest from the .hic file. The interaction profile of a specific $5-\mathrm{kb}$ or $10-\mathrm{kb}$ bin containing the anchor was then plotted in $R$. Replicate reproducibility was visualized with the mean profile shown as a line and the shading surrounding the mean representing the s.d. between replicates. For the HCASMC data, we observed low read coverage for allele-specific v4Cs at loci of interest. This is due to the density of SNPs for this genotype and a low number of reads containing a phased SNP. We thus could not observe interaction profiles when visualizing separate replicates with s.d. We therefore used pseudoreplicates for the HCASMC v4C visualizations ${ }^{52}$.

High-confidence Juicer loop calls were loaded into the WashU Epigenome Browser along with corresponding ATAC-seq profiles and publically available H3K27ac ChIP-seq data from the Roadmap Epigenomics Project. Browser shots from WashU track sessions were then included in $\mathrm{v} 4 \mathrm{C}$ and interaction map anecdotes. 


\section{Differential analysis of HiCCUPS loop calls}

Juicer loop calls from the three $\mathrm{T}$ cell subtypes were initially combined into a union set of $\mathrm{T}$ cell loops. Loop signal was then obtained for the biological replicates of each $\mathrm{T}$ cell subtype. Vanilla coverage square root (VCsqrt) normalized signal for the interaction matrix of each biological replicate using the Juicebox tools dump command. Normalized signal was then assigned to the union loop set in each replicate.

VCsqrt signal per sample was quantile-quantile normalized under the assumption that overall signal was identically distributed across all samples. Following normalization, samples for naive $\mathrm{T}, \mathrm{T}_{\mathrm{H}} 17$ and $\mathrm{T}_{\text {reg }}$ cells had Pearson correlations of $0.938,0.942$, and 0.934 , respectively. Principal-component analysis (PCA) was performed using the prcomp function in R, which demonstrated that the first principal component, which exhibited nearly identical loadings across the six samples, explained $93 \%$ of the variance across the six samples. This was taken to represent the shared signal across cell types. Principal components $2-4$ explained $2.2 \%, 2.0 \%$, and $1.4 \%$, respectively.

To study cell-type-specific looping, the residual signal per loop was taken after projecting the loop onto the unit vector along the diagonal (equal signal per cell type). Cell-typespecific and differential looping analysis were performed with the top and bottom 5\% of the distributions of either residual signal or differences between cell type residual signals. Hierarchical clustering was performed using the union of all differential loops in these extremes and using 1 minus the Pearson correlation as the distance metric. Quantilequantile plots were generated by permuting residuals from the same cell type or individual and summing them and using this distribution to calculate $P$ values for the observed sums.

In parallel, differential loops were called using edgeR for both the mouse ES and T cell data sets. Again, biological replicate loop signal was obtained across a union set of Juicer loops. We then used edgeR to identify loops with significant changes in signal among pairwise comparisons (FDR $<0.1, \log _{2}$ (fold change) $>1$ ). Notably, inspection of differential loops identified from the two methods showed high concordance.

Gene density was calculated from Ensembl gene annotations. GC content was calculated per $10-\mathrm{kb}$ bin using the BEDTools nuc function and aggregated as needed. Notably, Spearman correlation between the gene density of an entire chromosome and the number of differential loop anchors $(\rho=0.914)$ was much higher than the correlations between the variance in cell type signal per loop anchor and number of genes per 10-kb window $(\rho=0.322)$ and between differential loop anchors and gene density per 100-kb section $(\rho=0.083)$. Correlations between GC content and number of differential loops were similar at both the chromosome ( $\rho=0.729)$ and $100-\mathrm{kb}(\rho=0.148)$ levels, but, while local GC content is likely to confound relative abundance, it is unclear how chromosome-wide GC content could have the same effect.

For mouse ES cell analysis of H3K27ac- and cohesin-mediated HiChIP, we performed edgeR to obtain the biased loops for each factor. To determine the functional bias of the top loops, overlap was determined between edgeR differential loop anchors and relevant ChIPseq peaks. SMC1A ChIP-seq peaks were obtained from a published data set ${ }^{53}$. CTCF, RNA 
polymerase II, and H3K27ac ChIP-seq peaks were obtained from the mouse ENCODE repository ${ }^{54}$.

\section{RNA expression analysis}

Previously generated RNA-seq data ${ }^{55}$ from naive $\mathrm{T}, \mathrm{T}_{\mathrm{H}} 17$ and $\mathrm{T}_{\text {reg }}$ cells were downloaded as fastq files from ArrayExpress. Illumina adaptors were trimmed using CutAdapt, and Ensembl cDNA transcripts were quantified using kallisto. Sleuth was used to identify transcripts that were differentially expressed across cell types with FDR controlled at 5\%. The mean TPM was calculated per cell type, and TSS differential looping quantiles at genes with nonzero expression were correlated with differential expression quantiles of the same genes. Only 10-kb segments of the genome that contained a single annotated gene were considered to avoid errors in attribution of looping signal per 10-kb bin. For genes with multiple annotated TSSs, the 10-kb bin corresponding to the median TSS was used. Significance was assessed by the cor.test function in R.

\section{Distance-matched eQTL SNP-TSS comparisons}

We obtained three groups of eQTL SNP-TSS pairs within $1 \mathrm{Mb}$ for HiChIP EIS comparisons. The treatment group contained $\mathrm{CD} 4^{+} \mathrm{T}$ cell eQTL-TSS targets. We had two distance-matched groups as controls. The first control group contained $\mathrm{CD} 4^{+} \mathrm{T}$ cell eQTL SNP-random TSS pairs such that the distance between the eQTL SNP and random TSS differed by at most $5 \mathrm{~kb}$ with the treatment group. The second control group contained liver eQTL SNP-TSS targets that were also distance matched with the treatment group. The random eQTL SNP-TSS pairs were generated by individual chromosome, such that the numbers of control pairs and treatment pairs were the same for every chromosome. In total, there were 158,482 distance-matched eQTL-TSS pairs. We compared the 5-kb-resolution EIS values among the three eQTL SNP-TSS groups for all three T cell subtypes. Results showed that, in all cases, the EIS values between CD4 ${ }^{+} \mathrm{T}$ cell eQTL-TSS targets were significantly higher than the two control groups $\left(P<1 \times 10^{-16}\right.$, Kolmogorov-Smirnov test $)$.

\section{Distance-matched fine-mapped SNP-TSS comparisons}

We obtained a list of putatively causal SNPs from the PICS SNP list ${ }^{25}$ (PICS probability > 0.5 ), as well as fine-mapped SNPs associated with $\mathrm{IBD}^{38}$ or T1D ${ }^{39}$. Next, we obtained all SNPs in LD with each putatively causal SNP using European LD blocks determined by all SNPs with $r^{2} \geq 0.8$ with the SNP being considered. For the fine-mapped (T1D/IBD) sets, using SNPs in LD with highly significant GWAS SNPs might mean that there are several SNPs of equal or greater significance in the control set, but we still expect enrichment relative to the LD block.

We collected all the synthetic pairs between the putatively causal immune disease-related SNPs (IBD, T1D, and PICS) and nearby genes within $300 \mathrm{~kb}$. To perform the distancematched EIS comparisons, for each fine-mapped SNP category, we selected the SNP-TSS control pairs that satisfied two constraints: (i) the selected control SNP was positioned at least $5 \mathrm{~kb}$ away from the fine-mapped SNP in the same LD block and (ii) the distance of the SNP-TSS control pair differed with the fine-mapped SNP and the target gene by at most 5 $\mathrm{kb}$. 


\section{SNP-TSS loop analyses}

We obtained 7,747 PICS SNPs that are associated with autoimmune disease or non-immune traits $^{25} .4,331(55.9 \%)$ were associated with autoimmune disease, and 3,416 (44.1\%) were associated with non-immune traits. In addition, we obtained a set of SNPs associated with six overlapping autoimmune diseases using the GRASP catalog (genome-wide significance $\left.P<1 \times 10^{-8}\right)$.

We constructed a synthetic loop set for immune and non-immune SNPs and any TSS within $1 \mathrm{Mb}$ of each SNP. We then assigned VCsqrt signal in each biological replicate of the three T cell subtypes to the synthetic loop set, as described above.

VCsqrt signal per sample was quantile-quantile normalized as above. In this analysis, we did not restrict to HiCCUPS-identified loops but instead examined all possible interactions between a SNP and a TSS within $1 \mathrm{Mb}$. Many of these interactions do not exist and therefore had little or no matrix-balanced signal supporting them. While we removed all SNP-TSS pairs below an average of 1 normalized read per sample from subsequent analyses, in general, these false interactions contributed little to the overall differential signal for a trait.

H3K27ac data were downloaded from the WashU Roadmap repository. PICS SNPs were taken from Farh et al. $^{25}$. Rather than requiring strict membership within H3K27ac peaks, PICS SNPs were labeled as active if they were within $8 \mathrm{~kb}$ of a peak, increasing the number of nominally functional SNPs from $\sim 700$ to $\sim 3,200$ per cell type, out of 7,735 total candidate SNPs.

Differential looping across cell types was assessed by one-sided $t$ test per trait and activity partition if there were at least eight PICS SNP-TSS pairs in the partition. $\mathrm{T}_{\mathrm{H}} 17$ bias was defined as $\mathrm{T}_{\mathrm{H}} 17$ total loop signal minus naive $\mathrm{T}$ cell total loop signal; $\mathrm{T}_{\text {reg }}$ bias was defined as $\mathrm{T}_{\text {reg }}$ total loop signal minus naive $\mathrm{T}$ cell total loop signal; and naive $\mathrm{T}$ cell bias was defined as naive $T$ cell total loop signal minus one-half times the $\mathrm{T}_{\mathrm{H}} 17$ and $\mathrm{T}_{\text {reg }}$ total loop signals. For Supplementary Figure 12a,b,d, naive $\mathrm{T}$ cell bias was assessed only using SNPs that were active in naive $T$ cells, $T_{H} 17$ bias from SNPs active in $T_{H} 17$ and not naive $T$ cells, and $\mathrm{T}_{\text {reg }}$ bias from SNPs active in $\mathrm{T}_{\text {reg }}$ and not naive $\mathrm{T}$ cells. $P$ values were corrected for multiple-hypothesis testing by the Holm method using the p.adjust function in R. Bias assessed from SNPs with the opposing cell type specificities (for example, naive $\mathrm{T}$ cell bias using SNPs active in $\mathrm{T}_{\mathrm{H}} 17$ and $\mathrm{T}_{\text {reg }}$ cells but not naive $\mathrm{T}$ cells) yielded no significant hits after correction.

ATAC-seq

Cells were isolated and subjected to ATAC-seq as previously described ${ }^{16}$. Briefly, 55,000 cells were pelleted, resuspended in $50 \mu \mathrm{l}$ of lysis buffer $(10 \mathrm{mM}$ Tris- $\mathrm{HCl}, \mathrm{pH}$ 7.4, $3 \mathrm{mM}$ $\mathrm{MgCl}_{2}, 10 \mathrm{mM} \mathrm{NaCl}, 0.1 \% \mathrm{NP}-40$ (Igepal CA-630)), and immediately centrifuged at 500 r.c.f. for $10 \mathrm{~min}$ at $4{ }^{\circ} \mathrm{C}$. The nuclei pellets were resuspended in $50 \mu \mathrm{l}$ of transposition buffer ( $25 \mu \mathrm{l}$ of $2 \times$ TD buffer, $22.5 \mu \mathrm{l}$ of distilled water, $2.5 \mu \mathrm{l}$ of Illumina Tn5 transposase) and incubated at $37^{\circ} \mathrm{C}$ for $30 \mathrm{~min}$. Transposed DNA was purified with the MinElute PCR Purification kit (Qiagen), and eluted in $10 \mu \mathrm{l}$ of EB buffer. 


\section{ATAC-seq data processing}

Adaptor sequence trimming using SeqPurge and mapping to hg19 using Bowtie2 were performed. The reads were then filtered for mitochondrial reads, low-quality reads, and PCR duplicates. The filtered reads for each sample were merged, and peak calling was performed by MACS2. The reads in peaks for each individual sample were quantified using BEDTools intersect with the MACS2 narrow peaks. Peak counts were then combined into an $N \times M$ matrix where $N$ represents called peaks, $M$ represents the samples, and each value $D_{i, j}$ represents the peak intensity for respective peak $i$ in sample $j$. This matrix was then normalized using the 'CQN' package in R to minimize bias in GC content and length.

\section{CRISPRi validation of HiChIP targets}

For virus production, $5 \times 10^{6} \mathrm{HEK} 293 \mathrm{~T}$ cells were plated per $10-\mathrm{cm}$ plate. The following day, plasmid encoding lentivirus was cotransfected with pMD2.G and psPAX2 into the cells using Lipofectamine 3000 (Thermo Fisher, L3000) according to the manufacturer's instructions. Supernatant containing viral particles was collected $48 \mathrm{~h}$ after transfection and filtered. For lentivirus encoding individual sgRNAs, virus was concentrated tenfold using Lenti-X concentrator (Clontech, 631232) and stored at $-80{ }^{\circ} \mathrm{C}$.

To generate a My-La cell line expressing CRISPRi, $2 \times 10^{6}$ My-La cells were plated per T75 flask. A dCas9-BFP-KRAB-2A-Blast construct was generated by inserting a 2A-Blast cassette into dCas9-BFP-KRAB (Addgene, 46911). $24 \mathrm{~h}$ after plating, lentivirus harboring the dCas9-KRAB construct was added with polybrene $(4 \mu \mathrm{g} / \mathrm{ml})$. The medium was changed $24 \mathrm{~h}$ after infection and then again $48 \mathrm{~h}$ after infection with blasticidin (Thermo Fisher, A1113903) at a $4 \mu \mathrm{g} / \mathrm{ml}$ concentration. Blasticidin-resistant cells were selected for $8 \mathrm{~d}$ with the medium changed every other day.

Three different U6 sequences were used for transcription of three different sgRNAs targeting the candidate enhancers, as previously described ${ }^{56}$. For the $M Y C$ locus CRISPRi experiments, each enhancer was targeted by one guide, and all MYC GM or My-La enhancers together were therefore targeted in one experiment. For the PICS SNP CRISPRi experiments, three guides were targeted to a single SNP-containing enhancer. One of three sgRNAs was cloned into a lentiviral vector with a human (pMJ117, Addgene, 85997), mouse (pMJ179, Addgene, 85996), or bovine (pMJ114, Addgene, 85995) U6 promoter. These U6-sgRNA constructs were then combined into a lentivirus with a Puromycin-2AmCherry vector, which was modified from Addgene 46914. My-La-CRISPRi cells were infected with lentivirus harboring three sgRNAs and selected by puromycin (Thermo Fisher, A11138) at a final concentration of $1 \mu \mathrm{g} / \mathrm{ml}$. Previously reported sgRNAs targeting VPS54 or SEC24C were used for validating CRISPRi functionality in the My-La cell line ${ }^{57}$.

For readout of CRISPRi validation, we performed qRT-PCR and cell growth assays on three biological and two technical replicates. For qRT-PCR, RNA was extracted with TRIzol (Thermo Fisher, 15596026) and purified using the Zymo RNA Clean and Concentrator kit (Zymo Research, R1016). qRT-PCR was performed with Brilliant qRT-PCR Mastermix (Agilent, 600825). Ct values were measured using a LightCycler 480 instrument (Roche), and the relative expression level was calculated by the $\Delta \Delta \Delta \mathrm{Ct}$ method in comparison to a 
GAPDH control. Primer sequences are listed in Supplementary Table 6. For cell growth, we used the CellTiter-Glo kit (Promega, G7572) according to the manufacturer's instructions. Statistics for both RNA and cell growth changes were calculated using a Student's $t$ test against the non-targeting control.

\section{CRISPRa validation of HiChIP targets}

Jurkat cells were transduced with a lentiviral dCas9-VP64-2A-GFP expression vector (Addgene, 61422). Single $\mathrm{GFP}^{+}$cells were sorted by FACS into the wells of a 96-well plate, and a clone with bright, uniform GFP expression were selected for use in future experiments.

sgRNAs were cloned in arrayed format for $C D 69 \mathrm{HiChIP}$ peaks falling outside the range of the tiling CRISPRa screen ${ }^{26}$. sgRNAs were chosen on the basis of high predicted on-target activity ${ }^{58}$ and low predicted off-target activity ${ }^{59}$. sgRNAs were cloned into the lentiviral expression vector pCRISPRia-v2 (Addgene, 84832) as described in Horlbeck et al. ${ }^{60}$. Lentivirus was produced by transfecting HEK293T cells with standard packaging vectors using TransIT-LTI Transfection Reagent (Mirus, MIR 2306). The medium was changed $24 \mathrm{~h}$ after transfection. Viral supernatant was harvested at 48 and $72 \mathrm{~h}$ following transfection and immediately used for infection of Jurkat-dCas9-VP64 cells.

Jurkat-dCas9-VP64 cells were infected with lentiviral sgRNAs by resus-pending cells in a 1:1 mix of fresh medium and lentiviral supernatant at a final concentration of $0.25 \times 10^{6}$ cells $/ \mathrm{ml}$ with $5 \mu \mathrm{g} / \mathrm{ml}$ polybrene. Cells were spinfected for $1 \mathrm{~h}$ at 1,000 r.c.f. at $32{ }^{\circ} \mathrm{C}$. The next day, half of the medium was removed and replaced with fresh lentiviral supernatant and the spinfection was repeated. The next day, the cells were resuspended in fresh medium with $1.5 \mu \mathrm{g} / \mathrm{ml}$ puromycin and cultured for $2 \mathrm{~d}$ to remove uninfected cells. For readout of CRISPRa validation, we performed qRT-PCR and FACS on two biological and two technical replicates. RNA extraction and qRT-PCR were performed as described above. Expression of $\mathrm{CD} 69$ on infected cells $\left(\mathrm{GFP}^{+} \mathrm{BFP}^{+}\right)$was analyzed by flow cytometry with an Attune NxT flow cytometer (Life Technologies). Statistics for both RNA- and protein-level changes were calculated with one-way ANOVA followed by a Dunnett's multiplecomparisons test against the non-targeting control.

Additional methods are provided in the Supplementary Note.

\section{Data availability}

Raw and processed data are available at the Gene Expression Omnibus (GEO) under accession GSE101498. T cell ATAC-seq and HiChIP data sets can be visualized in the WashU Epigenome Browser with the following link: 98051079. A Life Sciences Reporting Summary is available.

\section{Supplementary Material}

Refer to Web version on PubMed Central for supplementary material. 


\section{Acknowledgments}

We thank members of the Chang and Greenleaf laboratories for helpful discussions and J. Tumey for artwork. We thank J. Engreitz, M. Pjanic, and C. Miller for assistance interpreting their published data sets. We thank X. Ji and J. Coller at the Stanford Functional Genomics Facility. We thank Agilent Technologies for generating oligonucleotide pools for cloning of the CRISPRa gRNAs. We thank the UC Berkeley High-Throughput Screening Facility and Flow Cytometry Facility. This work was supported by US National Institutes of Health (NIH) grants P50HG007735 (H.Y.C. and W.J.G.) U19AI057266 (W.J.G.), and 1UM1HG009436 (W.J.G.), the Human Frontier Science Program (W.J.G.), the Rita Allen Foundation (W.J.G.), and the Scleroderma Research Foundation (H.Y.C.). M.R.M. and E.A.B. acknowledge support from the National Science Foundation Graduate Research Fellowship. A.T.S. is a Cancer Research Institute Irvington Fellow supported by the Cancer Research Institute. B.G.G. was supported by an IGI-AstraZeneca Postdoctoral Fellowship. M.R.C. is supported by a grant from the Leukemia \& Lymphoma Society Career Development Program. J.E.C. was supported by the Li Ka Shing Foundation and the Heritage Medical Research Institute. W.J.G. and A.M. are Chan Zuckerberg Biohub investigators. Sequencing was performed by the Stanford Functional Genomics Facility (NIH S10OD018220).

\section{References}

1. Dixon JR, et al. Topological domains in mammalian genomes identified by analysis of chromatin interactions. Nature. 2012; 485:376-380. [PubMed: 22495300]

2. van Arensbergen J, van Steensel B, Bussemaker HJ. In search of the determinants of enhancerpromoter interaction specificity. Trends Cell Biol. 2014; 24:695-702. [PubMed: 25160912]

3. Lieberman-Aiden E, et al. Comprehensive mapping of long-range interactions reveals folding principles of the human genome. Science. 2009; 326:289-293. [PubMed: 19815776]

4. Rao SSP, et al. A 3D map of the human genome at kilobase resolution reveals principles of chromatin looping. Cell. 2014; 159:1665-1680. [PubMed: 25497547]

5. Fulco CP, et al. Systematic mapping of functional enhancer-promoter connections with CRISPR interference. Science. 2016; 354:769-773. [PubMed: 27708057]

6. Altshuler D, Daly MJ, Lander ES. Genetic mapping in human disease. Science. 2008; 322:881-888. [PubMed: 18988837]

7. Kumar V, et al. Human disease-associated genetic variation impacts large intergenic non-coding RNA expression. PLoS Genet. 2013; 9:e1003201. [PubMed: 23341781]

8. Edwards SL, Beesley J, French JD, Dunning AM. Beyond GWASs: illuminating the dark road from association to function. Am J Hum Genet. 2013; 93:779-797. [PubMed: 24210251]

9. Welter D, et al. The NHGRI GWAS Catalog, a curated resource of SNP-trait associations. Nucleic Acids Res. 2014; 42:D1001-D1006. [PubMed: 24316577]

10. Smemo S, et al. Obesity-associated variants within FTO form long-range functional connections with IRX3. Nature. 2014; 507:371-375. [PubMed: 24646999]

11. Claussnitzer M, et al. FTO obesity variant circuitry and adipocyte browning in humans. N Engl J Med. 2015; 373:895-907. [PubMed: 26287746]

12. Grubert F, et al. Genetic control of chromatin states in humans involves local and distal chromosomal interactions. Cell. 2015; 162:1051-1065. [PubMed: 26300125]

13. Mifsud B, et al. Mapping long-range promoter contacts in human cells with high-resolution capture Hi-C. Nat Genet. 2015; 47:598-606. [PubMed: 25938943]

14. Javierre BM, et al. Lineage-specific genome architecture links enhancers and non-coding disease variants to target gene promoters. Cell. 2016; 167:1369-1384. [PubMed: 27863249]

15. Sanjana NE, et al. High-resolution interrogation of functional elements in the noncoding genome. Science. 2016; 353:1545-1549. [PubMed: 27708104]

16. Mumbach MR, et al. HiChIP: efficient and sensitive analysis of protein-directed genome architecture. Nat Methods. 2016; 13:919-922. [PubMed: 27643841]

17. Heintzman ND, et al. Distinct and predictive chromatin signatures of transcriptional promoters and enhancers in the human genome. Nat Genet. 2007; 39:311-318. [PubMed: 17277777]

18. Creyghton MP, et al. Histone H3K27ac separates active from poised enhancers and predicts developmental state. Proc Natl Acad Sci USA. 2010; 107:21931-21936. [PubMed: 21106759] 
19. Rada-Iglesias A, et al. A unique chromatin signature uncovers early developmental enhancers in humans. Nature. 2011; 470:279-283. [PubMed: 21160473]

20. Ferraro A, et al. Interindividual variation in human T regulatory cells. Proc Natl Acad Sci USA. 2014; 111:E1111-E1120. [PubMed: 24610777]

21. Arvey A, et al. Genetic and epigenetic variation in the lineage specification of regulatory $\mathrm{T}$ cells. eLife. 2015; 4:e07571. [PubMed: 26510014]

22. Bettelli E, et al. Reciprocal developmental pathways for the generation of pathogenic effector $\mathrm{T}_{\mathrm{H}} 17$ and regulatory T cells. Nature. 2006; 441:235-238. [PubMed: 16648838]

23. Acosta-Rodriguez EV, et al. Surface phenotype and antigenic specificity of human interleukin 17producing T helper memory cells. Nat Immunol. 2007; 8:639-646. [PubMed: 17486092]

24. Morita R, et al. Human blood $\mathrm{CXCR} 5^{+} \mathrm{CD} 4^{+} \mathrm{T}$ cells are counterparts of $\mathrm{T}$ follicular cells and contain specific subsets that differentially support antibody secretion. Immunity. 2011;34:108121. [PubMed: 21215658]

25. Farh KKH, et al. Genetic and epigenetic fine mapping of causal autoimmune disease variants. Nature. 2015; 518:337-343. [PubMed: 25363779]

26. Simeonov DR, et al. Discovery of stimulation-responsive immune enhancers with CRISPR activation. Nature. 2017; 549:111-115. [PubMed: 28854172]

27. Engreitz JM, et al. Local regulation of gene expression by lncRNA promoters, transcription and splicing. Nature. 2016; 539:452-455. [PubMed: 27783602]

28. Dao LTM, et al. Genome-wide characterization of mammalian promoters with distal enhancer functions. Nat Genet. 2017; 49:1073-1081. [PubMed: 28581502]

29. Ernst J, Kellis M. ChromHMM: automating chromatin-state discovery and characterization. Nat Methods. 2012; 9:215-216. [PubMed: 22373907]

30. Sanborn AL, et al. Chromatin extrusion explains key features of loop and domain formation in wild-type and engineered genomes. Proc Natl Acad Sci USA. 2015; 112:E6456-E6465. [PubMed: 26499245]

31. Li G, et al. Extensive promoter-centered chromatin interactions provide a topological basis for transcription regulation. Cell. 2012; 148:84-98. [PubMed: 22265404]

32. Buenrostro JD, Giresi PG, Zaba LC, Chang HY, Greenleaf WJ. Transposition of native chromatin for fast and sensitive epigenomic profiling of open chromatin, DNA-binding proteins and nucleosome position. Nat Methods. 2013; 10:1213-1218. [PubMed: 24097267]

33. Samstein RM, et al. Foxp3 exploits a pre-existent enhancer landscape for regulatory $\mathrm{T}$ cell lineage specification. Cell. 2012; 151:153-166. [PubMed: 23021222]

34. Ye CJ, et al. Intersection of population variation and autoimmunity genetics in human $\mathrm{T}$ cell activation. Science. 2014; 345:1254665. [PubMed: 25214635]

35. Petukhova L, et al. Genome-wide association study in alopecia areata implicates both innate and adaptive immunity. Nature. 2010; 466:113-117. [PubMed: 20596022]

36. Raj T, et al. Polarization of the effects of autoimmune and neurodegenerative risk alleles in leukocytes. Science. 2014; 344:519-523. [PubMed: 24786080]

37. Schiering C, et al. The alarmin IL-33 promotes regulatory T-cell function in the intestine. Nature. 2014; 513:564-568. [PubMed: 25043027]

38. Huang $\mathrm{H}$, et al. Fine-mapping inflammatory bowel disease loci to single-variant resolution. Nature. 2017; 547:173-178. [PubMed: 28658209]

39. Onengut-Gumuscu S, et al. Fine mapping of type 1 diabetes susceptibility loci and evidence for colocalization of causal variants with lymphoid gene enhancers. Nat Genet. 2015; 47:381-386. [PubMed: 25751624]

40. O’Shea JJ, Lahesmaa R, Vahedi G, Laurence A, Kanno Y. Genomic views of STAT function in $\mathrm{CD}^{+}{ }^{+} \mathrm{T}$ helper cell differentiation. Nat Rev Immunol. 2011; 11:239-250. [PubMed: 21436836]

41. Miller CL, et al. Integrative functional genomics identifies regulatory mechanisms at coronary artery disease loci. Nat Commun. 2016; 7:12092. [PubMed: 27386823]

42. Nurnberg ST, et al. Coronary artery disease associated transcription factor TCF21 regulates smooth muscle precursor cells that contribute to the fibrous cap. PLoS Genet. 2015; 11:e1005155.

[PubMed: 26020946] 
43. McPherson R, et al. A common allele on chromosome 9 associated with coronary heart disease. Science. 2007; 316:1488-1491. [PubMed: 17478681]

44. Helgadottir A, et al. A common variant on chromosome 9p21 affects the risk of myocardial infarction. Science. 2007; 316:1491-1493. [PubMed: 17478679]

45. Clarke R, et al. Genetic variants associated with $\mathrm{Lp}(\mathrm{a})$ lipoprotein level and coronary disease. N Engl J Med. 2009; 361:2518-2528. [PubMed: 20032323]

46. CARDIoGRAMplusC4D Consortium. Large-scale association analysis identifies new risk loci for coronary artery disease. Nat Genet. 2013; 45:25-33. [PubMed: 23202125]

47. Leung D, et al. Integrative analysis of haplotype-resolved epigenomes across human tissues. Nature. 2015; 518:350-354. [PubMed: 25693566]

48. Dixon JR, et al. Chromatin architecture reorganization during stem cell differentiation. Nature. 2015; 518:331-336. [PubMed: 25693564]

49. Franzén O, et al. Cardiometabolic risk loci share downstream cis- and trans-gene regulation across tissues and diseases. Science. 2016; 353:827-830. [PubMed: 27540175]

50. Sinha, R., et al. Index switching causes "spreading-of-signal" among multiplexed samples in Illumina HiSeq 4000 DNA sequencing. Preprint at. bioRxiv. 2017. http://dx.doi.org/ $10.1101 / 125724$

51. Servant N, et al. HiC-Pro: an optimized and flexible pipeline for Hi-C data processing. Genome Biol. 2015; 16:259. [PubMed: 26619908]

52. Gjoneska E, et al. Conserved epigenomic signals in mice and humans reveal immune basis of Alzheimer's disease. Nature. 2015; 518:365-369. [PubMed: 25693568]

53. Kagey MH, et al. Mediator and cohesin connect gene expression and chromatin architecture. Nature. 2010; 467:430-435. [PubMed: 20720539]

54. Yue F, et al. A comparative encyclopedia of DNA elements in the mouse genome. Nature. 2014; 515:355-364. [PubMed: 25409824]

55. Bonnal RJP, et al. De novo transcriptome profiling of highly purified human lymphocytes primary cells. Sci Data. 2015; 2:150051. [PubMed: 26451251]

56. Adamson B, et al. A multiplexed single-cell CRISPR screening platform enables systematic dissection of the unfolded protein response. Cell. 2016; 167:1867-1882. [PubMed: 27984733]

57. Gilbert LA, et al. Genome-scale CRISPR-mediated control of gene repression and activation. Cell. 2014; 159:647-661. [PubMed: 25307932]

58. Doench JG, et al. Optimized sgRNA design to maximize activity and minimize off-target effects of CRISPR-Cas9. Nat Biotechnol. 2016; 34:184-191. [PubMed: 26780180]

59. Hsu PD, et al. DNA targeting specificity of RNA-guided Cas9 nucleases. Nat Biotechnol. 2013; 31:827-832. [PubMed: 23873081]

60. Horlbeck MA, et al. Nucleosomes impede Cas9 access to DNA in vivo and in vitro. eLife. 2016; 5:e12677. [PubMed: 26987018] 
a

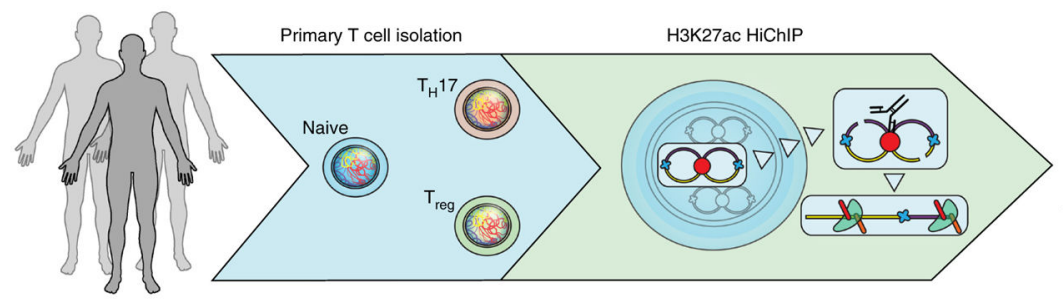

b
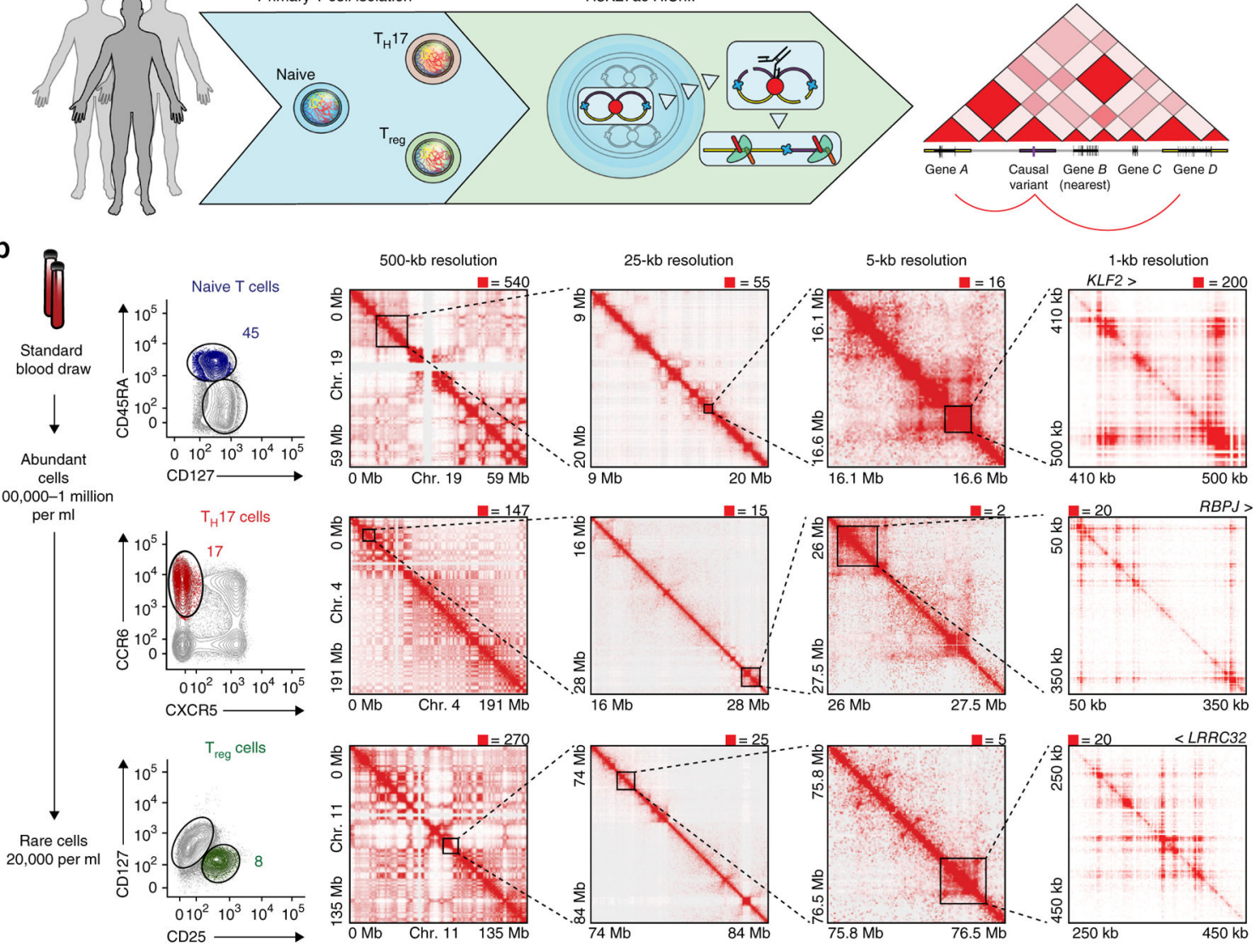

C
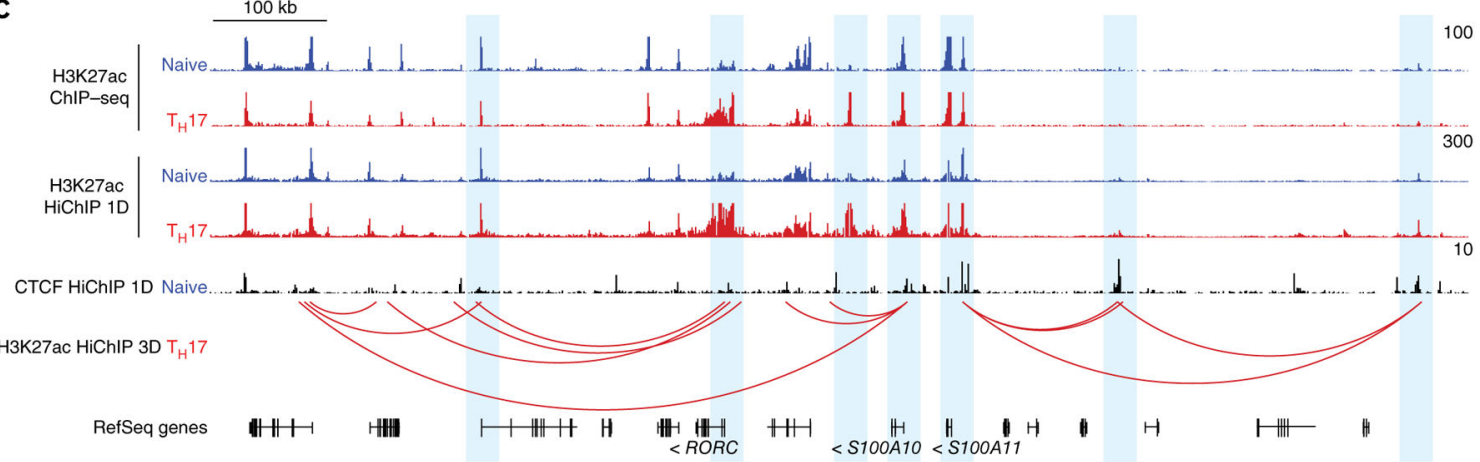

Figure 1.

HiChIP identifies high-resolution chromosome conformation in primary human T cells. (a)

Primary T cell H3K27ac HiChIP experimental outline. (b) Left, FACS strategy for naive,

$\mathrm{T}_{\mathrm{H}} 17$, and $\mathrm{T}_{\text {reg }}$ cells from total peripheral blood $\mathrm{CD} 4^{+} \mathrm{T}$ cells. Number represents the percentage of total $\mathrm{CD} 4^{+} \mathrm{T}$ cells within that gate. Right, Knight-Ruiz (KR) matrix-balanced interaction maps for naive, $\mathrm{T}_{\mathrm{H}} 17$, and $\mathrm{T}_{\text {reg }}$ cells at $500-\mathrm{kb}, 25-\mathrm{kb}$, and $5-\mathrm{kb}$ resolution, and raw interaction maps at 1-kb resolution, centered on the KLF2, RBPJ, and LRRC32 loci. (c) HiChIP 1D and 3D signal enrichment at the RORC locus in $\mathrm{T}_{\mathrm{H}} 17$ over naive $\mathrm{T}$ cells. 
a

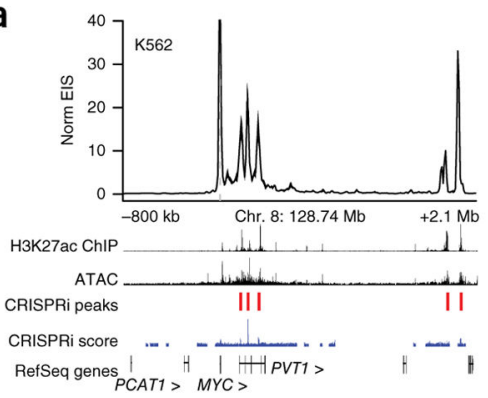

d

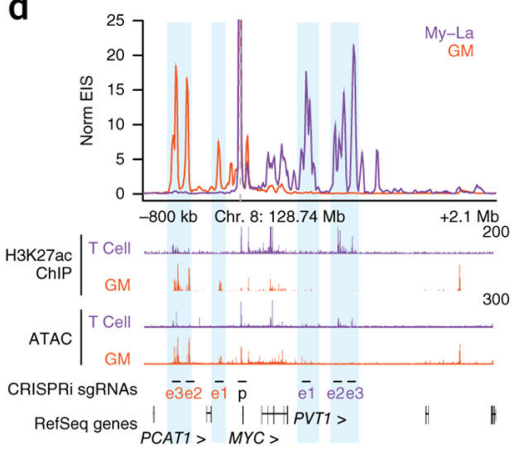

f

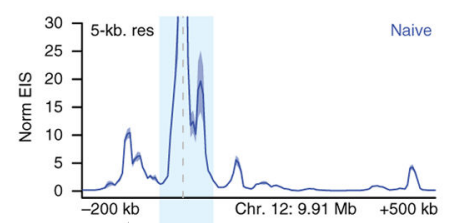

H3K27ac ChIP

ATAC

CRISPRa sgRNAs

RefSeq genes CLEC2D>

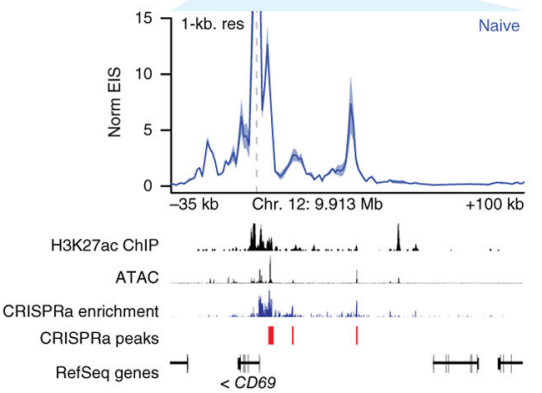

b
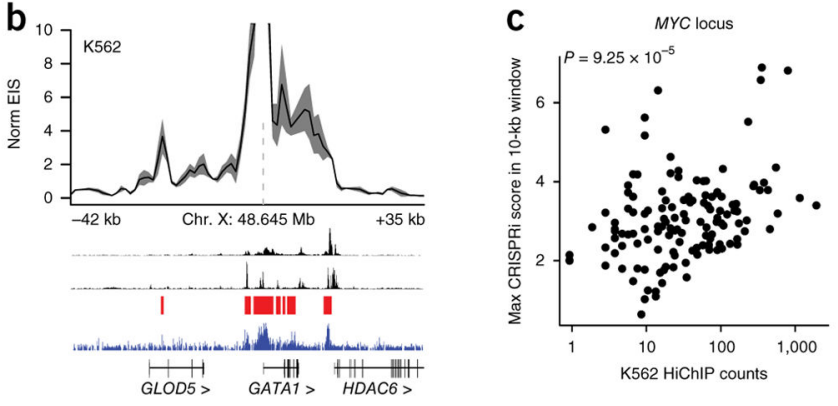

e

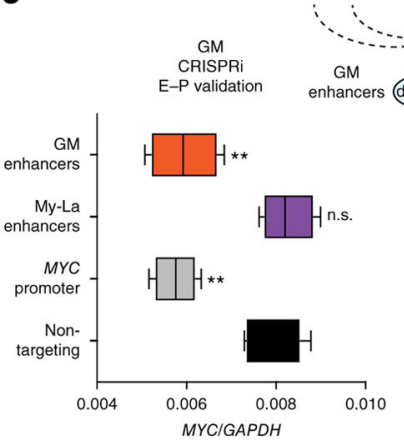

$\begin{array}{llll}\bar{e} & \bar{\theta} & \bar{e} & \bar{p}\end{array}$

$\overline{\mathrm{e} 1} \quad \overline{\mathrm{e} 2} \overline{\mathrm{e} 3}$
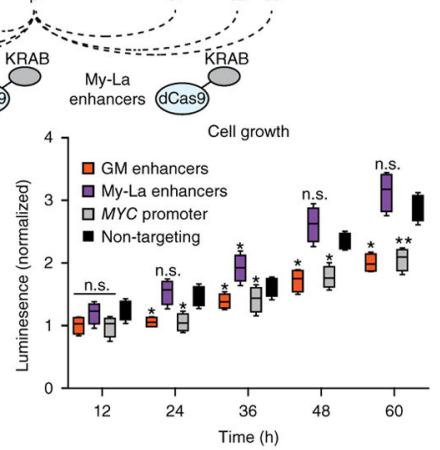

g
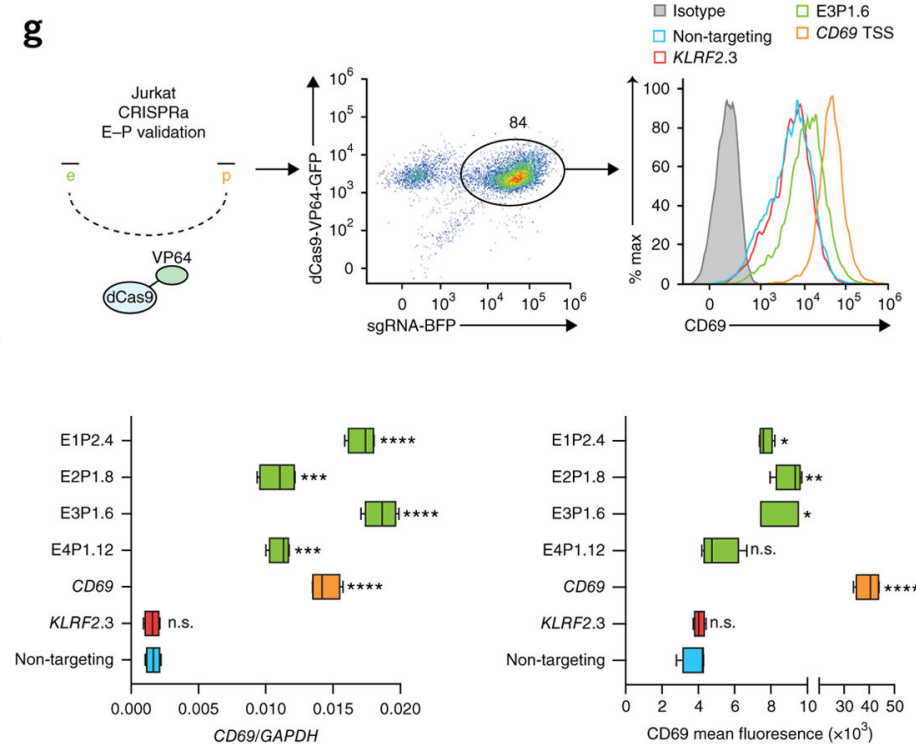

Figure 2.

Validation of regulatory elements identified by H3K27ac HiChIP with CRISPR interference and activation. (a) Interaction profile of the MYC promoter in K562 H3K27ac HiChIP at 10$\mathrm{kb}$ resolution. K562 H3K27ac ChIP-seq data are from the Encyclopedia of DNA Elements (ENCODE). CRISPRi-validated regulatory regions in K562 cells are indicated ${ }^{5}$. (b) Interaction profile of the GATA1 promoter in K562 H3K27 ac HiChIP at 1-kb resolution. CRISPRi-validated regulatory regions in K562 cells are indicated ${ }^{5}$. (c) Correlation of $M Y C$ K562 H3K27ac HiChIP signal with maximum CRISPRi score within the HiChIP 10-kb window. (d) Interaction profiles of the $M Y C$ promoter in GM and My-La H3K27ac HiChIP 
at 10-kb resolution. T cell H3K27ac ChIP-seq and ATAC-seq data are from naive T cells. (e) Top, CRISPRi validation in GM cells of GM- and My-La-biased MYC enhancers. E-P, enhancer-promoter. Bottom, $M Y C$ RNA levels by qRT-PCR and cell growth rates in CRISPRi GM cells with targeting of cell-type-biased enhancers, the $M Y C$ promoter, and a non-targeting negative control ( $n=3$ biological replicates, each with 2 technical replicates). (f) Interaction profile of the $C D 69$ promoter in Jurkat H3K27ac HiChIP at 5-kb and 1-kb resolution. The $1-\mathrm{kb}$ profile is focused on the window of the CRISPRa tiling screen. CRISPRa-validated regulatory regions in Jurkat cells are indicated ${ }^{26}$. (g) Top, CRISPRa validation in Jurkat cells of $C D 69$ distal enhancers. Bottom, $C D 69$ RNA and protein levels in CRISPRa Jurkat cells with targeting of distal enhancers, the $C D 69$ promoter, the $K L R F 2$ promoter as a locus negative control, and a non-targeting negative control ( $n=2$ biological replicates, each with 2 technical replicates). In the box plots, each box extends from the 25th to the 75th percentile with a line representing the median, and whiskers extend to the minimum and maximum values. $* P<0.05$; $* * P<0.01$; $* * * P<0.001$, **** $P<0.0001$ oneway ANOVA followed by Dunnett's multiple-comparisons test against the non-targeting control; n.s., not significant. 

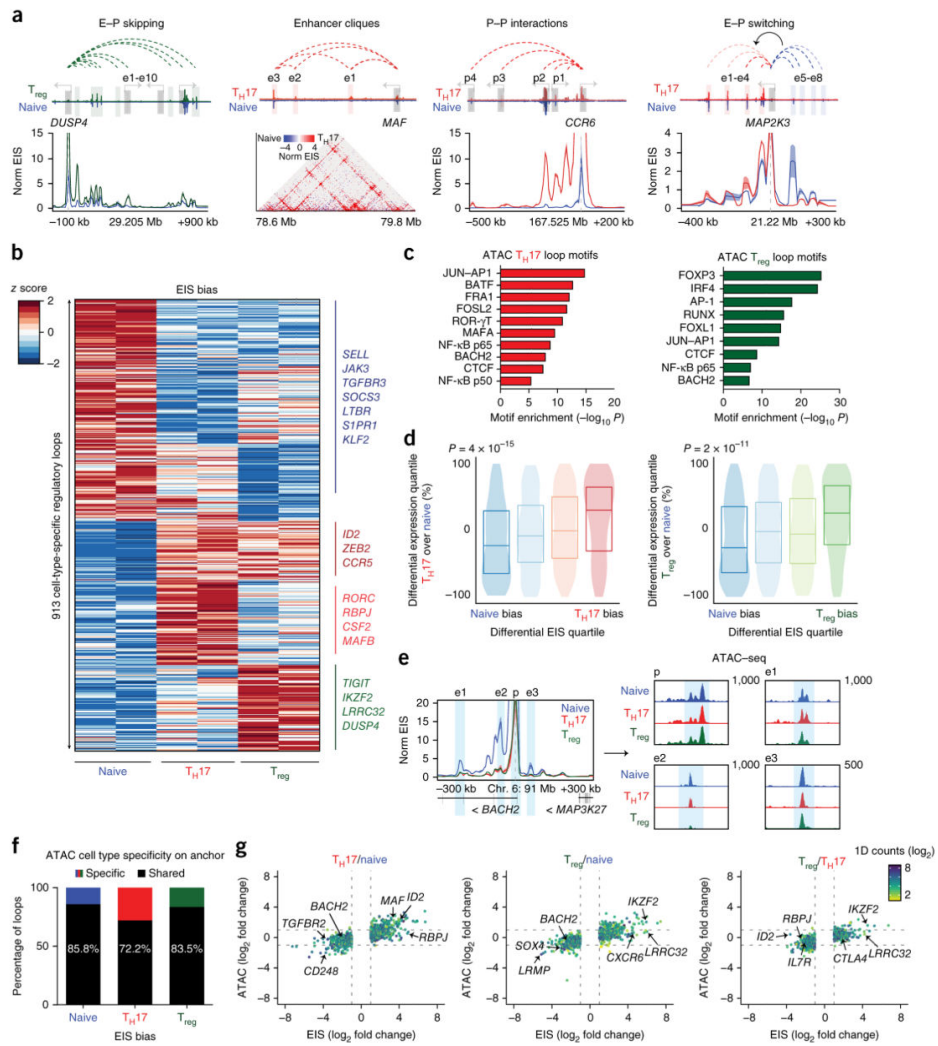

Figure 3.

Dynamic 3D enhancer landscapes in $\mathrm{T}$ cell differentiation. (a) Conformational features observed by H3K27ac HiChIP. (b) HiChIP EIS in 913 differential interactions identified in $\mathrm{T}$ cell subtypes. Interactions are clustered by cell type specificity. (c) Cell-type-specific motif identification from ATAC-seq peaks in biased EIS anchors. (d) EIS bias quartiles for naive to $T_{\mathrm{H}} 17$ and naive to $\mathrm{T}_{\text {reg }}$ differentiation, with corresponding differential RNA gene expression rankings. (e) Interaction profile of the $B A C H 2$ promoter at $5-\mathrm{kb}$ resolution, demonstrating shared accessibility signal at naive-biased EIS. (f) Proportion of ATAC-seq peaks within HiChIP differential interaction anchors that are cell type specific ( $\log _{2}$ (fold change) $>1$ ) or shared across all three subtypes. (g) Global correlation of EIS and ATACseq fold change in different pairwise comparisons of $\mathrm{T}$ cell subsets. 
a
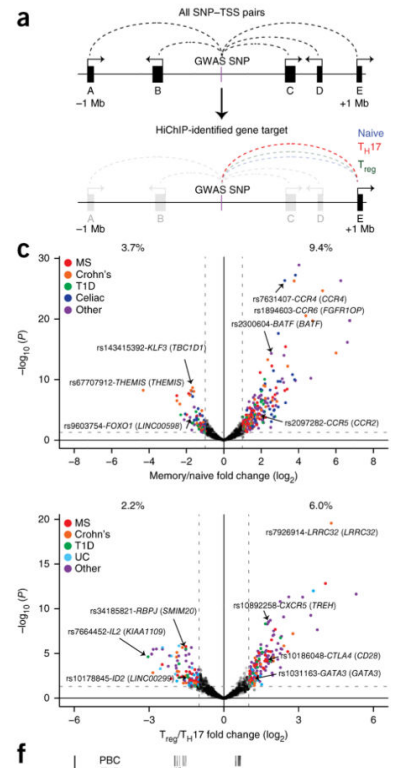

f
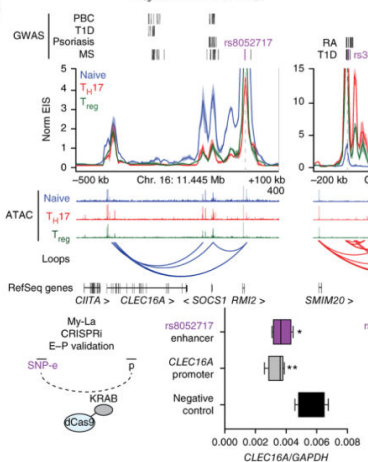

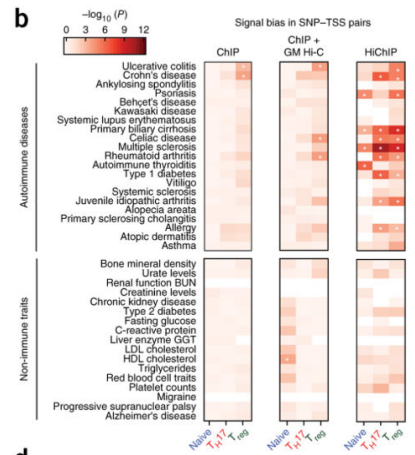

d

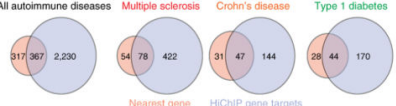

e

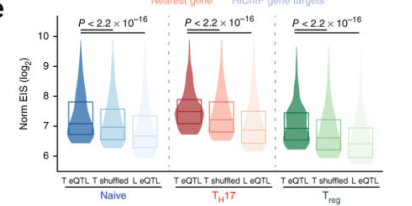

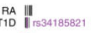

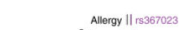

Figure 4.

HiChIP identifies cell type specificity and target genes of autoimmune disease-associated variants. (a) Generation of a loop set between all autoimmune disease SNPs and gene promoters within a 2-Mb region. (b) H3K27ac ChIP and HiChIP signal bias in T cell subtypes for SNP-TSS pairs. For each bin, PICS SNPs are tagged by H3K27ac only in the concordant cell type for the bias tested. SNPs are grossly divided into associations with autoimmune diseases or control, non-immune traits. Asterisks indicate FDR $<5 \%$. (c) EIS bias of SNP-TSS loops (with nearest gene annotated) in $\mathrm{T}_{\mathrm{H}} 17$ and $\mathrm{T}_{\text {reg }}$ subsets (memory $\mathrm{T}$ cells) versus naive $T$ cells and $T_{H} 17$ versus $T_{\text {reg }}$ cells. Percentages represent the percentage of SNP-TSS interactions that demonstrate biased HiChIP signal in the indicated cell type $\left(\log _{2}\right.$ (fold change) $>1$ ). MS, multiple sclerosis. (d) Number of HiChIP gene targets versus nearest-gene predictions for all looping non-genic autoimmune disease SNPs as well as SNPs for specific diseases. (e) Global validation of HiChIP SNP gene targets. Synthetic SNP-TSS pairs were generated from each $\mathrm{CD}^{+}{ }^{+}$eQTL SNP to its associated gene and compared to both a distance-matched shuffled SNP-TSS pair and a liver (L) eQTL SNPTSS pair. $P$ values were calculated by Kolmogorov-Smirnov test. (f) HiChIP target gene RNA levels by qRT-PCR in CRISPRi My-La cells with targeting of SNP-containing enhancers of interest, as well as positive-control sgRNAs to the HiChIP target promoters and a non-targeting negative control ( $n=3$ biological replicates, each with 2 technical 
replicates). RA, rheumatoid arthritis; $\mathrm{PBC}$, primary biliary cirrhosis. ${ }^{*} P<0.05 ; * * P<0.01$, two-tailed Student's $t$ test. In box plots, each box extends from the 25th to the 75th percentile with a line representing the median, and whiskers extend to the minimum and maximum values. 

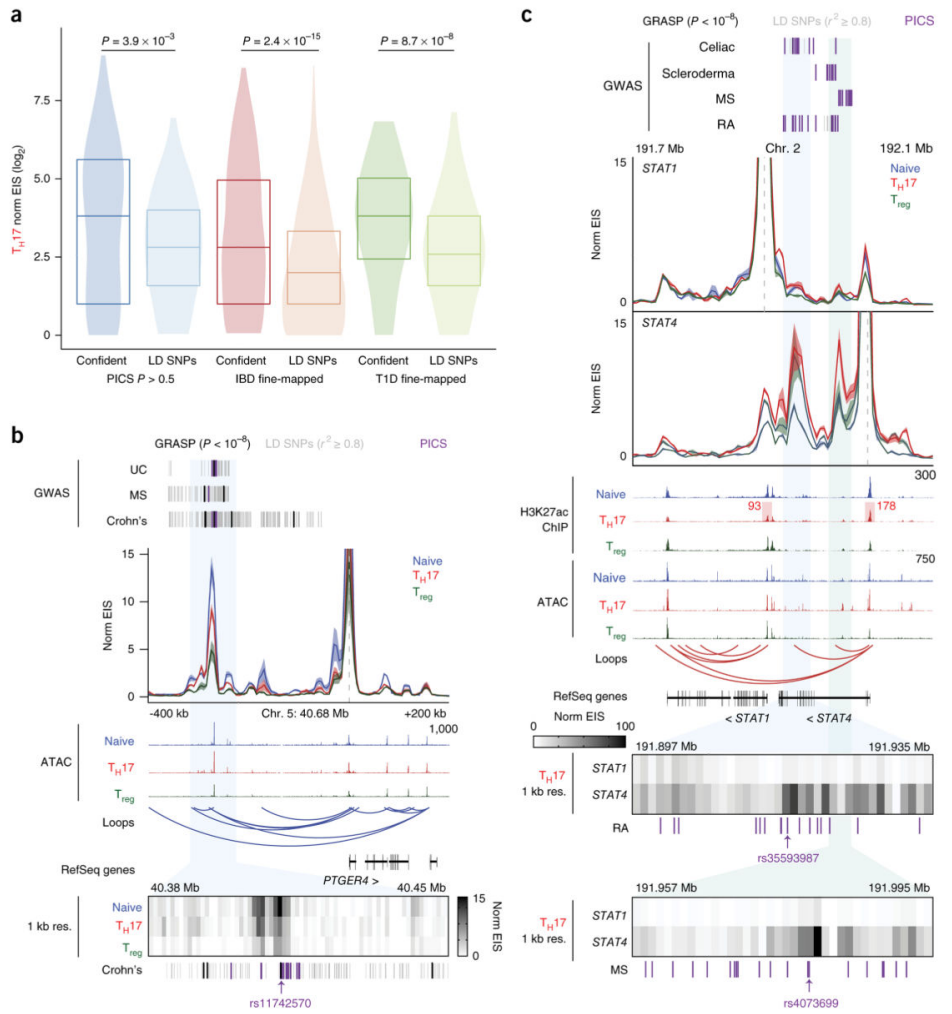

Figure 5.

Fine-mapping of GWAS-identified variants using H3K27ac HiChIP. (a) Global validation of HiChIP signal at putatively causal SNPs (confident) versus corresponding SNPs in LD (LD SNPs; $r^{2} \geq 0.8$ ) for $\mathrm{T}_{\mathrm{H}} 17$ cells. SNP-TSS pairs were generated from published fine-mapping data sets, in comparison to a distance-matched SNP-TSS pair set in the same LD block. $P$ values were calculated by Kolmogorov-Smirnov test. (b) Interaction profile of the PTGER4 promoter and a 1-kb-resolution visualization of the SNP-containing enhancer of interest. SNPs in LD $\left(r^{2} \geq 0.8\right)$ correspond to GRASP SNPs (genome-wide significance $P<1 \times$ $10^{-8}$ ). The highlighted SNP was identified in both the high-confidence PICS and GRASP data sets. UC, ulcerative colitis. (c) Interaction profiles of the STAT1 and STAT4 promoters, with 1-kb-resolution visualizations of the SNP-containing enhancers of interest. 1D signal contributions at the STAT1 and STAT4 promoters are highlighted. Highlighted SNPs are PICS SNPs closest to focal EIS for STAT4. 
a

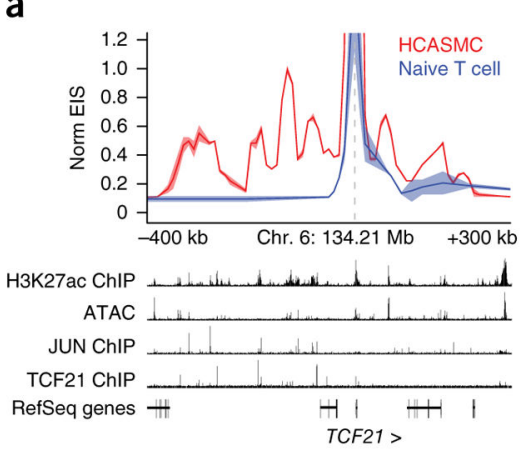

d

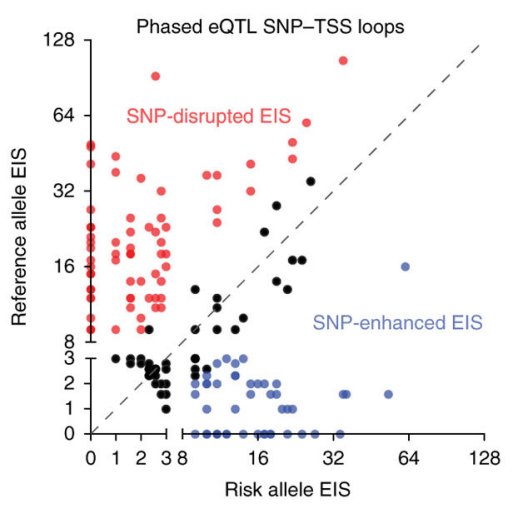

b

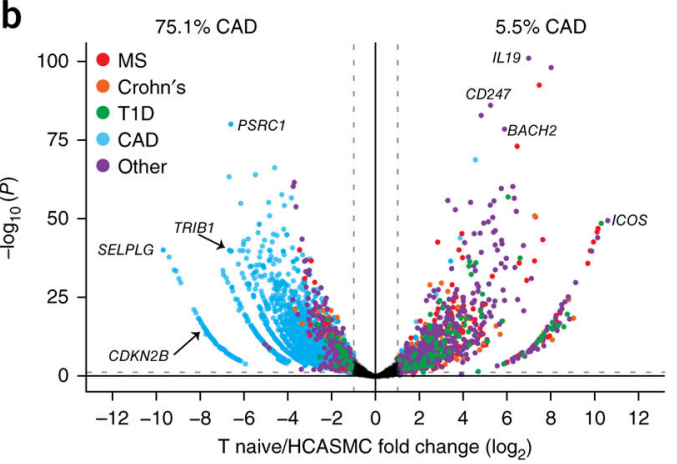

e

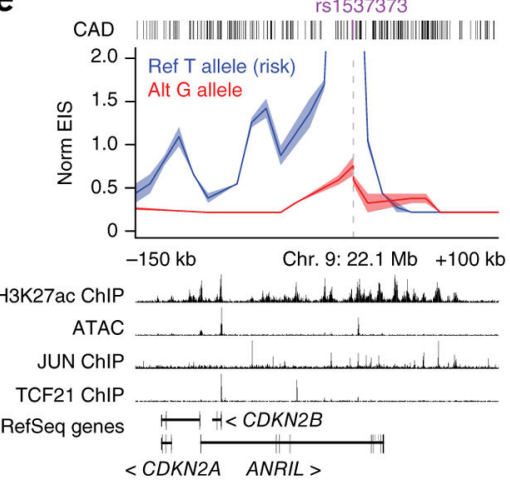

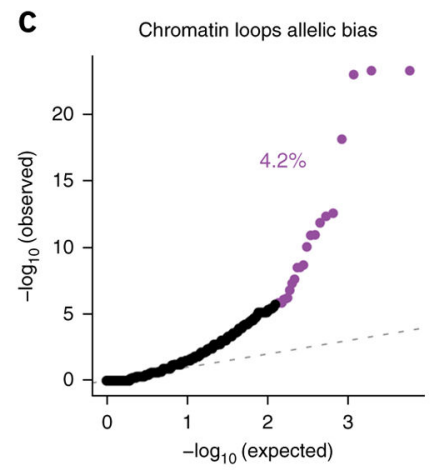

rs4562997
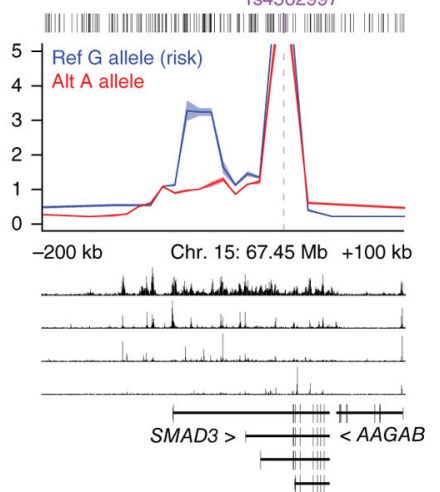

Figure 6.

HiChIP identifies allelic bias to target genes for cardiovascular disease risk variants. (a) Interaction profile of the TCF21 gene promoter for H3K27ac HiChIP of HCASMCs and naive T cells. (b) EIS bias between HCASMCs and naive T cells in a union set of CARDIoGRAMplusC4D CAD and PICS autoimmune disease SNP-TSS loops. Percentages represent the percent of SNP-TSS interactions that demonstrate biased HiChIP signal in the indicated cell type ( $\log 2$ (fold change) > 1). (c) Quantile-quantile plot of allelic EIS imbalance in high-confidence loops. Allelic mapping biased loops were identified through simulation and removed before EIS analysis. (d) EIS bias between CAD risk variants and their alternative alleles to eQTL-associated target genes. (e) Allele-specific HiChIP interaction profiles at the 9p21.3 and $S M A D 3$ loci at $10-\mathrm{kb}$ resolution to examine the functional consequence of a risk variant as compared to its alternative allele. ATAC-seq and ChIP-seq data sets shown are from ref. 41 . 